\title{
A New Extended Cosine-G Distributions for Lifetime Studies
}

\author{
Mustapha Muhammad ${ }^{1,2}$, Rashad A. R. Bantan ${ }^{3}$, Lixia Liu ${ }^{1, *}$, Christophe Chesneau ${ }^{4, *}$, Muhammad H. Tahir ${ }^{5}(0$, \\ Farrukh Jamal ${ }^{5}$ and Mohammed Elgarhy ${ }^{6}[$
}

1 School of Mathematical Sciences, Hebei Normal University, Shijiazhuang 050024, China; mmmahmoud12@sci.just.edu.jo or mmuhammad.mth@buk.edu.ng

2 Department of Mathematical Sciences, Bayero University Kano, Kano 700241, Nigeria

3 Department of Marine Geology, Faculty of Marines Science, King AbdulAziz University, Jeddah 21551, Saudi Arabia; rbantan@kau.edu.sa

4 Department of Mathematics, Université de Caen, LMNO, Campus II, Science 3, 14032 Caen, France

5 Department of Statistics, The Islamia University of Bahawalpur, Punjab 63100, Pakistan; mht@iub.edu.pk (M.H.T.); farrukh.jamal@iub.edu.pk (F.J.)

6 The Higher Institute of Commercial Sciences, Al Mahalla Al Kubra, Algarbia 31951, Egypt; m_elgarhy85@sva.edu.eg

* Correspondence: liulixia@hebtu.edu.cn (L.L.); christophe.chesneau@gmail.com or christophe.chesneau@unicaen.fr (C.C.)

Citation: Muhammad, M.; Bantan, R.A.R.; Liu, L.; Chesneau, C.; Tahir, M.H.; Jamal, F.; Elgarhy, M. A New Extended Cosine-G Distributions for Lifetime Studies. Mathematics 2021, 9, 2758. https://doi.org/10.3390/ math9212758

Academic Editor: Vasile Preda

Received: 14 September 2021

Accepted: 26 October 2021

Published: 30 October 2021

Publisher's Note: MDPI stays neutral with regard to jurisdictional claims in published maps and institutional affiliations.

Copyright: (c) 2021 by the authors. Licensee MDPI, Basel, Switzerland. This article is an open access article distributed under the terms and conditions of the Creative Commons Attribution (CC BY) license (https:// creativecommons.org/licenses/by/ $4.0 /)$.

\begin{abstract}
In this article, we introduce a new extended cosine family of distributions. Some important mathematical and statistical properties are studied, including asymptotic results, a quantile function, series representation of the cumulative distribution and probability density functions, moments, moments of residual life, reliability parameter, and order statistics. Three special members of the family are proposed and discussed, namely, the extended cosine Weibull, extended cosine power, and extended cosine generalized half-logistic distributions. Maximum likelihood, least-square, percentile, and Bayes methods are considered for parameter estimation. Simulation studies are used to assess these methods and show their satisfactory performance. The stress-strength reliability underlying the extended cosine Weibull distribution is discussed. In particular, the stress-strength reliability parameter is estimated via a Bayes method using gamma prior under the square error loss, absolute error loss, maximum a posteriori, general entropy loss, and linear exponential loss functions. In the end, three real applications of the findings are provided for illustration; one of them concerns stress-strength data analyzed by the extended cosine Weibull distribution.
\end{abstract}

Keywords: trigonometric distributions; moments; entropy; maximum likelihood estimation; leastsquare estimation; percentile estimation; Bayes estimation; stress-strength parameter

\section{Introduction}

In various practical analyses, the choice of an appropriated statistical model is crucial. This is particularly problematic for some data analyses for which the commonly used distributional models are incapable of providing a satisfactory fit. In the past few years, some solutions have been proposed, including the construction of families of flexible distributions. The majority of them seek to improve the functionality of a baseline distribution by employing various mathematical generalization (or extension) techniques. Thus, constructed, the corresponding models may contain some additional parameters which confer enough flexibility to explore real-life data in various fields of studies, such as reliability, survival analysis, computer sciences, finance, biomedical sciences, medicine, hydrology, etc.

Most of the extensions of the classical distributions generated are usually algebraic, but recent attention has been given to statistical distributions based on trigonometric functions. The trigonometric functions can play a vital role in statistical studies with unlimited motivations and influence. These can be a means of extending models by the convolution 
of algebraic-based and trigonometric-based distributions. The proposed statistical distributions based on trigonometric functions could provide researchers with more alternatives in terms of the availability of distributions having trigonometric functions. Furthermore, they might lead to additional new problems that allow more exploration of computational techniques, simulation, and algorithms. The following are some statistical distributions, or families of distributions, based on trigonometric functions: Fisher (F) distribution by [1], Von Mises (VM) distribution by [2], beta-trigonometric (BT) distribution by [3], circular Cauchy (CC) distribution by [4], sine square (SS) distribution by [5], sine-generated (SG) family by [6,7], cosine-generated (CSG) family by [8], new cosine-sine-generated (NCSG) family by [9], transformed sine-generated (TSG) family by [10], new sine-generated (NSG) family by [11], tan-generated (TG) family by [12], polyno-expo-trigonometric (PET) family by [13], sin Topp-Leone-generated (STLG) family by [14], sine extended odd Fréchetgenerated (SEOFG) family by [15], new exponential with trigonometric function (NET) distribution by [16], weighted cosine exponential distribution by [17], arc-sine distribution, and raised cosine distribution, among others. In particular, the SG, CSG, NSG, TG, STLG, and SEOFG families benefit from the oscillating nature of trigonometric functions to enrich the modeling capabilities of any chosen baseline distribution.

In this paper, we enhance the aforementioned idea by introducing the extended cosine-generated (ECSG) family of distributions. As a main motivation, it provides a simple one-parameter extension of the SG family, improving its theoretical and statistical possibilities. As a short list of improvements, we can mention more flexible functional shapes, tail characteristics, kurtosis, and skewness. Thus, the ECSG family can be useful for a variety of applications. With this in mind, this work develops both the practical side and the crucial theoretical results of the proposed family in an extensive manner.

The rest of the paper is arranged as follow: In Section 2, the ECSG family is presented in detail, along with proof of some important properties and descriptions of special members of interest. In Section 3, various parameter estimation and simulation studies are given. Section 4 concerns the real data illustration of the findings. In Section 5, some conclusions are provided.

\section{The ECSG Family}

\subsection{Presentation}

The generalization of classical distributions proposed by [18] allowed us to introduce one additional parameter to the existing distribution in an exponentiated fashion. This technique is widely known in the literature. In our study, we considered an alternative way of the exponentiation technique that, combined with the SG family, gives the ECSG family of distributions. The details are given below.

Let $s(x ; \xi)$ be the survival function (sf) related to a baseline cumulative distribution function (cdf) of a continuous distribution, denoted by $G(x ; \xi)$, with a vector of parameters $\xi$. That is, $s(x ; \xi)=1-G(x ; \xi)$. Let $g(x ; \xi)$ be the pdf corresponding to $G(x ; \xi)$. Then, the cdf of the ECSG family with parameter $\alpha>0$ is given by:

$$
F(x)=1-\left[1-\cos \left(\frac{\pi}{2} s(x ; \xi)\right)\right]^{\alpha},
$$

and the corresponding pdf is indicated as:

$$
f(x)=\alpha \frac{\pi}{2} g(x ; \xi) \sin \left(\frac{\pi}{2} s(x ; \xi)\right)\left[1-\cos \left(\frac{\pi}{2} s(x ; \xi)\right)\right]^{\alpha-1} .
$$

In full generality, functions $F(x)$ and $f(x)$ characterize the entire ECSG family in the probabilistic sense. Since $\cos ((\pi / 2)(1-x))=\sin ((\pi / 2) x)$, we had:

$$
\cos \left(\frac{\pi}{2} s(x ; \xi)\right)=\sin \left(\frac{\pi}{2} G(x ; \xi)\right),
$$


which corresponds to the cdf of the SG family. For the purposes of this study, it was, however, more practical to work with the sf of the baseline distribution instead of its cdf. Some motivations and remarks on the ECSG family are formulated below:

- We could write $F(x)=K[\cos ((\pi / 2) s(x ; \xi))]$, where $K(u)=1-\left(1-u^{\beta}\right)^{\alpha}$ is the cdf of the Kumaraswamy distribution with $\beta=1$ (see [19]). This exponentiation technique is an alternative to the one of [18].

- Clearly, when $\alpha=1$, the ECSG family was reduced to the SG family.

- The pdf of the ECSG family is a weighted version of the pdf of the SG family; indeed, if we denoted the pdf of the SG family by $f_{*}(x)$, we could write:

$$
f(x)=w_{\alpha}(x) f_{*}(x),
$$

where $w_{\alpha}(x)=\alpha[1-\cos ((\pi / 2) s(x ; \xi))]^{\alpha-1}$. We, thus, saw how the parameter $\alpha$ modulated the functionalities of $w_{\alpha}(x)$, and the strong relationship existing with the respective pdfs. Naturally, the presence of the weight function $w_{\alpha}(x)$ completely modified the analytical structure of $f(x)$; the mode and tail properties of $f_{\alpha}(x)$ truly depend on the variations of $w_{\alpha}(x)$.

- When $\alpha$ is a positive integer, $F(x)$ is the cdf of the random variable $Y_{\alpha}=\min \left(X_{1}, \ldots, X_{\alpha}\right)$, where $X_{1}, \ldots, X_{\alpha}$ are independent random variables with the cdf of the $S G$ family. Thus, the ECSG family has an interpretation in terms of the distribution of minimum of several random variables.

- The function $F(x)$ increased with respect to $\alpha$. This implies a first-order stochastic dominance with the SG family, which differs in nature according to $\alpha \in(0,1)$ and $\alpha \geq 1$.

- $\quad$ More secondary, $\operatorname{since} \sin (x)^{2}=[1-\cos (2 x)] / 2$, we could rewrite $F(x)$ as:

$$
F(x)=1-2^{\alpha}\left[\sin \left(\frac{\pi}{4} s(x ; \xi)\right)\right]^{2 \alpha} .
$$

As main reliability functions, the sf, hazard rate function (hrf), and cumulative hrf (chrf) of the ECSG family were given as:

$$
\begin{aligned}
& R(x)=\left[1-\cos \left(\frac{\pi}{2} s(x ; \xi)\right)\right]^{\alpha} \\
& h(x)=\frac{\alpha(\pi / 2) g(x ; \xi) \sin ((\pi / 2) s(x ; \xi))}{1-\cos ((\pi / 2) s(x ; \xi))}
\end{aligned}
$$

and

$$
T(x)=-\alpha \log \left[1-\cos \left(\frac{\pi}{2} s(x ; \xi)\right)\right]
$$

respectively.

The cdf, pdf, and hrf are involved in many modeling and statistical tools and, thus, were of particular interest in the rest of the study.

Following the structure of the studies in $[9,11,12]$, based on the functions above, the rest of the theoretical study focused on some asymptotic results, quantile function, a series representation of the cdf and pdf, moments, moments of residual life, reliability parameter, and order statistics.

\subsection{Asymptotic Study}

The following lemma allowed us to understand the asymptotic behavior of the cdf, pdf, and hrf according to the asymptotic behavior of $s(x ; \xi)$.

Lemma 1. The following equivalence results held: 
On the one hand, as $s(x ; \xi) \rightarrow 1$, we had:

$$
\begin{aligned}
& F(x) \sim \alpha \frac{\pi}{2}(1-s(x ; \xi)), \\
& f(x) \sim h(x) \sim \alpha \frac{\pi}{2} g(x ; \xi) .
\end{aligned}
$$

On the other hand, as $s(x ; \xi) \rightarrow 0$, we had:

$$
\begin{aligned}
& F(x) \sim 1-\frac{\pi^{2 \alpha}}{8^{\alpha}} s(x ; \xi)^{2 \alpha}, \\
& f(x) \sim 2 \alpha \frac{\pi^{2 \alpha}}{8^{\alpha}} g(x ; \xi) s(x ; \xi)^{2 \alpha-1}, \\
& h(x) \sim 2 \alpha \frac{g(x ; \xi)}{s(x ; \xi)} .
\end{aligned}
$$

The role of $\alpha$ on the asymptotic behavior of the main functions of the ECSG family was now clarified; it had a constant effect when $s(x ; \xi) \rightarrow 0$, and an exponentiated effect when $s(x ; \xi) \rightarrow 1$, except on $h(x)$ when the effect was constant. As $s(x ; \xi) \rightarrow 0$, we saw that $h(x)$ was equivalently proportional to the hrf of the baseline distribution.

\subsection{Quantile Function}

Let $q_{G}(u)$ be the quantile function (qf) corresponding to $G(x ; \xi)$. The qf of the ECSG family can be presented as:

$$
Q(u)=q_{G}\left(1-\frac{2}{\pi}\left[\arccos \left(1-(1-u)^{1 / \alpha}\right)\right]\right), \quad 0 \leq u \leq 1 .
$$

In particular, $M=Q(1 / 2)$ is the median. The qf served as a means of model parameter estimation, and was used in generating random data. In addition, the qf was used in computations of some distribution properties and goodness-of-fit measures. The qf of a distribution could be used to analyze its skewness and kurtosis by means of Bowley's skewness and Moor's kurtosis, defined as:

$$
B=\frac{Q(3 / 4)-2 Q(2 / 4)+Q(1 / 4)}{Q(3 / 4)-Q(1 / 4)}, \quad M=\frac{Q(7 / 8)-Q(5 / 8)+Q(3 / 8)-Q(1 / 8)}{Q(6 / 8)-Q(2 / 8)},
$$

respectively.

\subsection{Functional Series Representation}

2.4.1. For the cdf

We provided a series representation of the cdf of the ECSG family. In this regard, we needed to use several existing series expansion results. First, for $|z| \leq 1$ and $b>0$ that we supposed to be a non-integer, we had:

$$
(1-z)^{b}=1+\sum_{i=1}^{\infty}(-1)^{i}\left(\begin{array}{l}
b \\
i
\end{array}\right) z^{i}
$$

where $\left(\begin{array}{l}b \\ i\end{array}\right)$ refers to the generalized binomial coefficient. In addition, the following trigonometric representations held:

$$
\cos x=\sum_{n=0}^{\infty} \frac{(-1)^{n} x^{2 n}}{(2 n) !}, \quad \sin x=\sum_{n=0}^{\infty} \frac{(-1)^{n} x^{2 n+1}}{(2 n+1) !} .
$$

As the last important known result, for a power series raised to the power of a positive integer, we had the representation provided by [20], and recalled it in the next lemma. 
Lemma 2 (Established by [20]). For a given power series of the form $\sum_{k=0}^{\infty} a_{k} x^{k}$ and a positive integer $n$, we had:

$$
\left(\sum_{k=0}^{\infty} a_{k} x^{k}\right)^{n}=\sum_{k=0}^{\infty} c_{k} x^{k}
$$

where $c_{0}=a_{0}^{n}, c_{m}=\left[1 /\left(m a_{0}\right)\right] \sum_{k=1}^{m}(k n-m+k) a_{k} c_{m-k}$ for $m \geq 1$.

By applying Equation (4), the cdf of the ECSG family could be represented as:

$$
F(x)=1-\left[1-\cos \left(\frac{\pi}{2} s(x ; \xi)\right)\right]^{\alpha}=\sum_{k=1}^{\infty} w_{k}(\alpha)\left[\cos \left(\frac{\pi}{2} s(x ; \xi)\right)\right]^{k}
$$

where $w_{k}(\alpha)=(-1)^{k+1}\left(\begin{array}{c}\alpha \\ k\end{array}\right)$. It followed from the expansion of the cosine function in Equation (5), that:

$$
F(x)=\sum_{k=1}^{\infty} w_{k}(\alpha)\left[\sum_{n=0}^{\infty} a_{n} s(x ; \xi)^{2 n}\right]^{k}
$$

where $a_{n}=(-1)^{n}(\pi / 2)^{2 n} /[(2 n) !]$. By applying Lemma 2 to the above equation, we obtained:

$$
F(x)=\sum_{k=1}^{\infty} \sum_{n=0}^{\infty} w_{k}(\alpha) c_{n} s(x ; \xi)^{2 n},
$$

where $c_{0}=a_{0}^{k}, c_{m}=\left[1 /\left(m a_{0}\right)\right] \sum_{n=1}^{m}(n k-m+n) a_{n} c_{m-n}$ for $m \geq 1$. Thus,

$$
F(x)=\sum_{k=1}^{\infty} \sum_{n=0}^{\infty} b_{k, n} s(x ; \xi)^{2 n}
$$

where $b_{k, n}=w_{k}(\alpha) c_{n}$. From the approximation point-of-view, the following approximation involving finite sums was valid:

$$
F(x) \approx \sum_{k=1}^{v} \sum_{n=0}^{v} b_{k, n} s(x ; \xi)^{2 n}
$$

where $v$ is a large integer that operates on sums truncation.

\subsubsection{For the pdf}

Now, we expressed the pdf of the ECSG family in a series form. We started by applying Equations (4) and (5) to obtain:

$$
\left[1-\cos \left(\frac{\pi}{2} s(x ; \xi)\right)\right]^{\alpha-1}=1+\sum_{i=1}^{\infty}(-1)^{i}\left(\begin{array}{c}
\alpha-1 \\
i
\end{array}\right)\left[\sum_{n=0}^{\infty} a_{n} s(x ; \xi)^{2 n}\right]^{i}
$$

where $a_{n}=(-1)^{n}(\pi / 2)^{2 n} /[(2 n) !]$. By Lemma 2, we obtained:

$$
\left[1-\cos \left(\frac{\pi}{2} s(x ; \xi)\right)\right]^{\alpha-1}=1+\sum_{i=1}^{\infty} \sum_{n=0}^{\infty} d_{i, n} s(x ; \xi)^{2 n}
$$

where $d_{i, n}=(-1)^{i}\left(\begin{array}{c}\alpha-1 \\ i\end{array}\right) c_{n}, c_{0}=a_{0}^{i}, c_{m}=\left[1 /\left(m a_{0}\right)\right] \sum_{n=1}^{m}(n i-m+n) a_{n} c_{m-n}$ for $m \geq 1$.

Hence, by putting the above expression in $f(x)$ and using the sine series representation in Equation (5), we obtained: 


$$
f(x)=\alpha \frac{\pi}{2} g(x ; \xi) \sum_{l=0}^{\infty} \frac{(-1)^{l}(\pi / 2)^{2 l+1}}{(2 l+1) !} s(x ; \xi)^{2 l+1}\left[1+\sum_{i=1}^{\infty} \sum_{n=0}^{\infty} d_{i, n} s(x ; \xi)^{2 n}\right] .
$$

Thus, the pdf of the ECSG family could be represented as:

$$
f(x)=\sum_{l=0}^{\infty} \psi_{l}^{(1)} g(x ; \xi) s(x ; \xi)^{2 l+1}+\sum_{i=1}^{\infty} \sum_{l, n=0}^{\infty} \psi_{i, l, n}^{(2)} g(x ; \xi) s(x ; \xi)^{2(l+n)+1},
$$

where $\psi_{l}^{(1)}=\alpha(-1)^{l}(\pi / 2)^{2 l+2} /[(2 l+1) !]$, and $\psi_{i, l, n}^{(2)}=\psi_{l}^{(1)} d_{i, n}$. This expansion result would be useful for the moment analysis. Again, from the approximation point-of-view, we could reduced the "sophisticated" function $f(x)$ by the following approximation:

$$
f(x) \approx \sum_{l=0}^{v} \psi_{l}^{(1)} g(x ; \xi) s(x ; \xi)^{2 l+1}+\sum_{i=1}^{v} \sum_{l, n=0}^{v} \psi_{i, l, n}^{(2)} g(x ; \xi) s(x ; \xi)^{2(l+n)+1},
$$

where $v$ is a large integer that operates on sums truncation.

For some baseline distributions, the series expansions were more practical when they involved the exponentiated $\operatorname{cdf} G(x ; \xi)$. In this case, with the application of the standard binomial formula, we could expand the pdf to:

$$
f(x)=\sum_{l=0}^{\infty} \sum_{w=0}^{2 l+1} \psi_{l, w}^{*(1)} g(x ; \xi) G(x ; \xi)^{w}+\sum_{i=1}^{\infty} \sum_{l, n=0}^{\infty} \sum_{w=0}^{2(n+l)+1} \psi_{i, l, n, w}^{*(2)} g(x ; \xi) G(x ; \xi)^{w}
$$

where $\psi_{l, w}^{*(1)}=(-1)^{w}\left(\begin{array}{c}2 l+1 \\ w\end{array}\right) \psi_{i}^{(1)}$ and $\psi_{i, l, n, w}^{*(2)}=(-1)^{w} \underset{w}{(\underset{w}{2(n+l)+1})} \psi_{i, l, n}^{(2)}$.

\subsection{Moments}

The moment analysis of the ECSG family was now under investigation. Let $X$ be a random variable with a certain distribution from the ECSG family. Then, the $r^{\text {th }}$ moment of $X$ was classically defined by $\mu_{r}=E\left[X^{r}\right]=\int_{-\infty}^{\infty} x^{r} f(x) d x$. That is,

$$
\mu_{r}=\alpha \frac{\pi}{2} \int_{-\infty}^{\infty} x^{r} g(x ; \xi) \sin \left(\frac{\pi}{2} s(x ; \xi)\right)\left[1-\cos \left(\frac{\pi}{2} s(x ; \xi)\right)\right]^{\alpha-1} d x,
$$

which could be expressed as the following integral over the unit interval:

$$
\mu_{r}=\int_{0}^{1}\left[q_{G}\left(1-\frac{2}{\pi}\left[\arccos \left(1-(1-u)^{1 / \alpha}\right)\right]\right)\right]^{r} d u
$$

The above expressions may not have had a closed-form solution; thus, they could be computed numerically via software such as $R$, MATLAB, and Mathematica. By using the series representation in Equation (6), $\mu_{r}$ could be expressed as:

$$
\mu_{r}=\sum_{l=0}^{\infty} \psi_{l}^{(1)} v_{r, 2 l+1}+\sum_{i=1}^{\infty} \sum_{l, n=0}^{\infty} \psi_{i, l, n}^{(2)} v_{r, 2(l+n)+1}
$$

where $v_{r, w}=\int_{-\infty}^{\infty} x^{r} g(x ; \xi) s(x ; \xi)^{w} d x$. Coefficient $v_{r, w}$ was calculable in a scholarly manner for most of the standard distributions.

From an alternative point of view, the following series representation followed from Equation (7):

$$
\mu_{r}=\sum_{l=0}^{\infty} \sum_{w=0}^{2 l+1} \frac{\psi_{l, w}^{*(1)}}{w+1} \mu_{r, w}^{*}+\sum_{i=1}^{\infty} \sum_{l, n=0}^{\infty} \sum_{w=0}^{2(n+l)+1} \frac{\psi_{i, l, n, w}^{*(2)}}{w+1} \mu_{r, w}^{*}
$$


where $\mu_{r, w}^{*}$ is the $r^{\text {th }}$ moment of a random variable with the exponentiated baseline distribution defined with the power parameter $w+1$.

To complete the moment analysis of the ECSG family, we could also derive the $r^{\text {th }}$ incomplete moment of $X$ defined as $I_{r}(t)=\int_{-\infty}^{t} x^{r} f(x) d x$. That is,

$$
I_{r}(t)=\alpha \frac{\pi}{2} \int_{-\infty}^{t} x^{r} g(x ; \xi) \sin \left(\frac{\pi}{2} s(x ; \xi)\right)\left[1-\cos \left(\frac{\pi}{2} s(x ; \xi)\right)\right]^{\alpha-1} d x,
$$

which could be expressed in an alternative manner as:

$$
I_{r}(t)=\int_{0}^{F(t)}\left[q_{G}\left(1-\frac{2}{\pi}\left[\arccos \left(1-(1-u)^{1 / \alpha}\right)\right]\right)\right]^{r} d u .
$$

We could establish series expansions of $I_{r}(t)$ via Equations (6) and (7) in the same way as for $\mu_{r}$.

The first incomplete moment $I_{1}(t)$ was derived from Equation (10) by taking $r=1$. It is an important measure; it is useful for obtaining the mean deviations of the mean and the mean deviations of the median defined by:

$$
\delta_{1}(X)=2 \mu_{1} F\left(\mu_{1}\right)-2 I_{1}\left(\mu_{1}\right), \quad \delta_{2}(X)=\mu_{1}-2 I_{1}(M),
$$

respectively. The first incomplete moment was also the main ingredient in the definitions of the Lorenz and Bonferroni curves, which are very useful and applicable in reliability, economics, demography, and insurance. The Lorenz and Bonferroni curves are defined by $L(p)=I_{1}(q) / \mu_{1}$ and $B(q)=I_{1}(q) /\left(p \mu_{1}\right)$, respectively, where $q=Q(p)$ from Equation (3).

Further, the first incomplete moment could be used to determine the two moments of residual life. The mean residual life and mean reverse residual life functions are vital tools in engineering, reliability, quality control, life testing, etc. They are also used in finding the asymptotic distributions of order statistics. By assuming $X$ as a lifetime distribution, the mean residual life of $X$ was defined as:

$$
T(t)=E[X-t \mid X>t]=\int_{0}^{\infty} \frac{R(x+t)}{R(t)} d x .
$$

The mean reverse residual life of $X$ was defined by:

$$
\bar{T}(t)=E[t-X \mid X \leq t]=\int_{0}^{t} \frac{F(x)}{F(t)} d x .
$$

The asymptotic behavior of $T(t)$ and $\bar{T}(t)$ are useful in extreme value theory, among others. For special members of the ECSG family, some results of this nature were proven.

\subsection{Reliability}

The study of a system's performance can be performed via a special parameter called the stress-strength parameter. It is very important in engineering practice to have a good design of a system in such a way that the strength is always greater than the assumed stress. The system is certain to fail if and only if the stress applied is greater than the strength. Suppose a component had stress modeled by a random variable $X$ and was subjected to a strength modeled by a random variable $Y$; then, $R_{s}=P(Y<X)$ would determine the system's performance and define the stress-strength parameter. Some applications of stress-strength reliability in various fields can be found in [21] (chap. 7). Parameter $R_{S}$ has been studied by many authors in the literature from various viewpoints. For example, for the study based on independent random variables $X$ and $Y$ that follow normal $(N)$ distributions see [22,23], exponential (E) distributions see [24], generalized logistic (GL) distributions see [25], Weibull (W) distributions see [26-28], generalized exponential (GE) distributions see [29], generalized exponential Poisson (GEP) distributions see [30], 
beta-Erlang truncated exponential (BETE) distributions see [31], Poisson-generalized halflogistic (PGHL) distributions see [32], and Poisson half-logistic (PHL) distributions see [33], among others.

Here, we derived the expression of $R_{S}$ by assuming $X$ and $Y$ had a distribution from the ECSG family with a common parameter vector $\xi$. More precisely, let $X$ be a random variable with pdf $f_{1}(x)$ defined as in Equation (2) with the parameters $\alpha_{1}$ and $\xi$, and $Y$ be a random variable with the cdf $F_{2}(x)$ defined as in Equation (1) with the parameters $\alpha_{2}$ and $\xi$. It was assumed that $X$ and $Y$ were independent. Then, the stress-strength reliability parameter was expressed as $R_{s}=\int_{-\infty}^{\infty} f_{1}(x) F_{2}(x) d x$. Therefore,

$$
R_{s}=1-\frac{\alpha_{1} \pi}{2} \int_{-\infty}^{\infty} g(x ; \xi) \sin \left(\frac{\pi}{2} s(x ; \xi)\right)\left[1-\cos \left(\frac{\pi}{2} s(x ; \xi)\right)\right]^{\alpha_{1}+\alpha_{2}-1} d x .
$$

By applying the change $u=1-\cos ((\pi / 2) s(x ; \xi))$, we immediately obtained:

$$
R_{s}=1-\alpha_{1} \int_{0}^{1} u^{\alpha_{1}+\alpha_{2}-1} d u=1-\frac{\alpha_{1}}{\alpha_{1}+\alpha_{2}}=\frac{\alpha_{2}}{\alpha_{1}+\alpha_{2}} .
$$

In this setting, parameter $R_{s}$ had a simple expression that could be used quite efficiently for various inferential purposes. This aspect was investigated later through a computational analysis.

\subsection{Order Statistics}

Let $\left(X_{1}, \ldots, X_{n}\right)$ be a random sample of size $n$ from a distribution of the ECSG family. Then, for $i=1, \ldots, n$, the pdf of the $i^{t h}$ order statistic, denoted by $f_{i: n}(x)$, could be expressed in the function of $R(x)$ as:

$$
\begin{aligned}
f_{i: n}(x) & =i\left(\begin{array}{c}
n \\
i
\end{array}\right) f(x) R(x)^{n-i}(1-R(x))^{i-1} \\
& =i\left(\begin{array}{c}
n \\
i
\end{array}\right) f(x) \sum_{j=0}^{i-1}\left(\begin{array}{c}
i-1 \\
j
\end{array}\right)(-1)^{j} R(x)^{n-i+j} .
\end{aligned}
$$

By noticing that $R(x)^{n-i+j}$ is the sf of the ESCG family with power parameter $\alpha(n-i+j)$, the pdf of the $i^{\text {th }}$ order statistic could be express as a mixture of pdfs of the ECSG family as:

$$
f_{i: n}(x)=\sum_{j=0}^{i-1} \tau_{i, j} f(x ; \alpha(n-i+j+1), \xi),
$$

where $\tau_{i, j}=\left[(-1)^{j} /(n-i+j+1)\right] i\left(\begin{array}{c}n \\ i\end{array}\right)\left(\begin{array}{c}i-1 \\ j\end{array}\right)$ and $f(x ; \alpha(n-i+j+1), \xi)$ are the pdfs of the ECSG family defined with power parameter $\alpha(n-i+j+1)$. The $r^{\text {th }}$ moments of the $i^{\text {th }}$ order statistic could be computed from Equation (11) by considering Equation (9) as:

$$
E\left[X_{i: n}^{r}\right]=\sum_{j=0}^{i-1} \tau_{i, j} \mu_{r, j}^{* *}
$$

where $\mu_{r, j}^{* *}$ is the $r^{\text {th }}$ moment of a random variable with a certain distribution of the ECSG family with power parameter $\alpha(n-i+j+1)$.

In a coming subsection, we considered the asymptotic distributions for the extreme order statistics, i.e., the minimum $X_{1: n}$ and maximum $X_{n: n}$, under some distributional assumptions on $X_{1}, \ldots, X_{n}$. For the details, see [34]. 


\subsection{Special Members of the ECSG Family}

Here, some special members of the ECSG family were derived and discussed. Namely, they were the extended cosine Weibull (ECSW), extended cosine power (ECSP), and extended cosine generalized half-logistic (ECSGHL) distributions.

\subsubsection{The ECSW Distribution}

The cdf, pdf, and hrf of the ECSW distribution were obtained by considering the baseline distribution of the $\mathrm{W}$ distribution with sf and pdf defined as $s(x ; \lambda, \theta)=e^{-\lambda x^{\theta}}$ and $g(x ; \lambda, \theta)=\lambda \theta x^{\theta-1} e^{-\lambda x^{\theta}}$, respectively, where $x, \lambda, \theta>0$. That is, for $x>0$, the cdf, pdf, and hrf of the ECSW distribution were:

$$
\begin{gathered}
F(x)=1-\left[1-\cos \left(\frac{\pi}{2} e^{-\lambda x^{\theta}}\right)\right]^{\alpha}, \\
f(x)=\frac{\alpha \lambda \theta \pi}{2} x^{\theta-1} e^{-\lambda x^{\theta}} \sin \left(\frac{\pi}{2} e^{-\lambda x^{\theta}}\right)\left[1-\cos \left(\frac{\pi}{2} e^{-\lambda x^{\theta}}\right)\right]^{\alpha-1},
\end{gathered}
$$

and

$$
h(x)=\frac{\alpha \lambda \theta(\pi / 2) x^{\theta-1} e^{-\lambda x^{\theta}} \sin \left((\pi / 2) e^{-\lambda x^{\theta}}\right)}{1-\cos \left((\pi / 2) e^{-\lambda x^{\theta}}\right)},
$$

respectively. These functions were all equal to 0 for $x<0$. In some parts of the study, this distribution was denoted as $\operatorname{ECSW}(\alpha, \theta, \lambda)$, to specify the parameters. As a result, the ECSW distribution was a new three-parameter lifetime distribution that aimed to improve on the W distribution's modeling capabilities. In order to illustrate this claim, Figure 1 shows the plots of the corresponding pdf and hrf. We can state that the pdf could decrease or be unimodal. Different curvature characteristics were observed, such as right-skewed and almost symmetric forms, along with different platenesses of the curves. The bathtub and reversed bathtub shapes were observed for the hrf, as well as increasing and decreasing shapes. These observations were in favor of the application of the ECSW model for the analysis of lifetime data of decreasing or right-skewed unimodal natures.
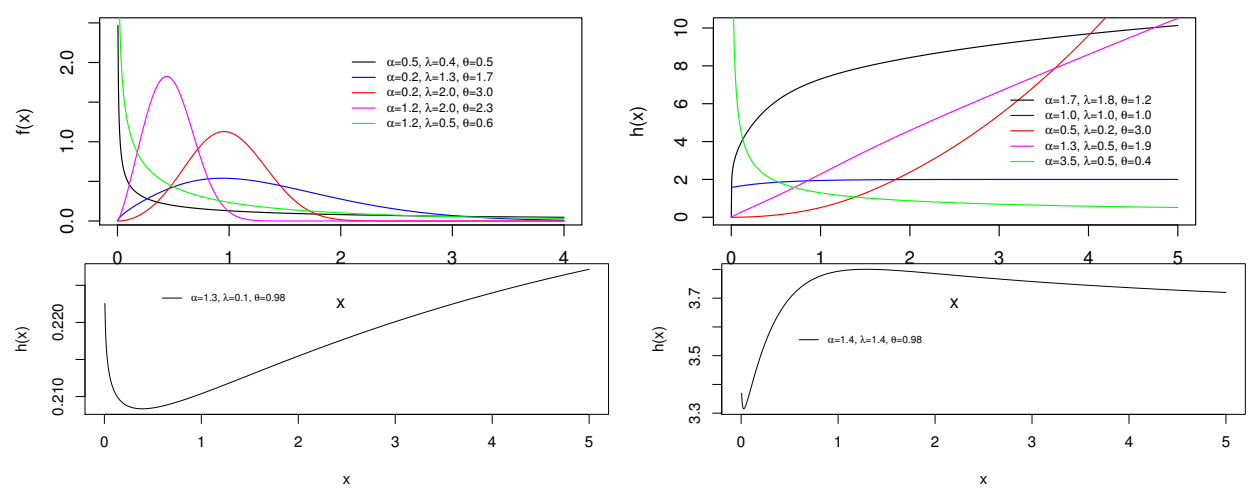

Figure 1. Plots of the pdf and hrf of the ECSW distribution for some parameter values.

It is worth noting that the Rmpfr Package in R provided by [35] was used to obtain high-precision numbers in the computation of term $[1-\cos ((\pi / 2) s(x ; \xi))]^{\alpha-1}$ in plots, depending on the value of $\alpha$.

All the general results stated for the ECSG family in the previous parts could be applied for the ECSW distribution. In particular, the quantile function of the ECSW distribution was given as: 


$$
Q(u)=\left\{-\frac{1}{\lambda} \log \left(\frac{2}{\pi}\left[\arccos \left(1-(1-u)^{1 / \alpha}\right)\right]\right)\right\}^{1 / \theta}, \quad 0 \leq u \leq 1
$$

Based on Equation (8), the $r^{\text {th }}$ moment of a random variable $X$ with the ECSW distribution could be expressed as:

$$
\mu_{r}=\frac{\alpha \lambda \theta \pi}{2} \int_{0}^{\infty} x^{r+\theta-1} e^{-\lambda x^{\theta}} \sin \left(\frac{\pi}{2} e^{-\lambda x^{\theta}}\right)\left[1-\cos \left(\frac{\pi}{2} e^{-\lambda x^{\theta}}\right)\right]^{\alpha-1} d x .
$$

The software R, MATLAB, and Mathematica could be used to calculate this integral. Alternatively, by using the series representation in Equation (6), $\mu_{r}$ could be expressed as:

$$
\mu_{r}=\sum_{l=0}^{\infty} \psi_{l}^{(1)} v_{r, 2 l+1}+\sum_{i=1}^{\infty} \sum_{l, n=0}^{\infty} \psi_{i, l, n}^{(2)} v_{r, 2(l+n)+1}
$$

where

$$
\begin{aligned}
v_{r, w} & =\int_{-\infty}^{\infty} x^{r} g(x ; \xi) s(x ; \xi)^{w} d x=\lambda \theta \int_{0}^{\infty} x^{r+\theta-1} e^{-(w+1) \lambda x^{\theta}} d x \\
& =\frac{1}{(w+1)^{r / \theta+1}} \frac{1}{\lambda^{r / \theta}} \Gamma\left(\frac{r}{\theta}+1\right),
\end{aligned}
$$

and $\Gamma(a)$ is the standard gamma function. A similar treatment could be applied for the incomplete moments, and the related functions.

The following theorem provides asymptotic results on the mean residual life and mean reverse residual life.

Theorem 1. Let $X$ be a random variable following the ECSW distribution. Then,

- As $t \rightarrow \infty$, the mean residual life of $X$ satisfies

$$
T(t) \sim \frac{e^{2 \alpha \lambda t^{\theta}}}{\theta(2 \alpha \lambda)^{1 / \theta}} \Gamma\left(\frac{1}{\theta}, 2 \alpha \lambda t^{\theta}\right),
$$

where $\Gamma(a, x)$ is the upper incomplete gamma function,

- As $t \rightarrow 0$, the mean reverse residual life of $X$ satisfies

$$
\bar{T}(t) \sim \frac{t}{\theta+1} .
$$

Proof. From Lemma 1, we could derive the asymptotic of the sf of the ECSW distribution as $t \rightarrow \infty$, from which we established that:

$$
T(t)=\int_{0}^{\infty} \frac{R(x+t)}{R(t)} d x \sim e^{2 \alpha \lambda t^{\theta}} \int_{0}^{\infty} e^{-2 \alpha \lambda(x+t)^{\theta}} d x .
$$

If we applied the change of variable $u=2 \alpha \lambda(x+t)^{\theta}$, we obtained:

$$
T(t) \sim \frac{e^{2 \alpha \lambda t^{\theta}}}{\theta(2 \alpha \lambda)^{1 / \theta}} \int_{2 \alpha \lambda t^{\theta}}^{\infty} u^{1 / \theta-1} e^{-u} d u=\frac{e^{2 \alpha \lambda t^{\theta}}}{\theta(2 \alpha \lambda)^{1 / \theta}} \Gamma\left(\frac{1}{\theta}, 2 \alpha \lambda t^{\theta}\right) .
$$

On the other hand, as $t \rightarrow 0$, from Lemma 1 , we could derive the asymptotic of the cdf of the ECSW distribution and obtain:

$$
\bar{T}(t)=\int_{0}^{t} \frac{F(x)}{F(t)} d x \sim \frac{1}{1-e^{-\lambda t^{\theta}}} \int_{0}^{t}\left(1-e^{-\lambda x^{\theta}}\right) d x \sim \frac{1}{t^{\theta}} \int_{0}^{t} x^{\theta} d x=\frac{t}{\theta+1} .
$$

This concludes the proof of Theorem 1. 
An application of Theorem 1 was given in the next result, which concerned the asymptotic distributions of the extreme ordered statistics.

Theorem 2. Let $\left\{X_{n}\right\}_{n}$ be a sequence of independent random variables with the ECSW distribution.

- Suppose that $\theta=1$. Let $B_{n}=\left(X_{n: n}-a_{n}\right) / b_{n}$ where $a_{n}=Q(1-1 / n)$ and $b_{n}=$ $E\left[X-a_{n} \mid X>a_{n}\right]$, then:

$$
\lim _{n \rightarrow \infty} P\left(B_{n} \leq x\right)=e^{-e^{-x}},
$$

for $x \in \mathbb{R}$. The cdf of the standard Gumbel distribution was obtained as limit; $\left\{B_{n}\right\}_{n}$ converged in distribution to a random variable with such a Gumbel distribution.

- Let $B_{n}^{*}=\left(X_{1: n}-a_{n}^{*}\right) / b_{n}^{*}$, where $a_{n}^{*}=0$ and $b_{n}^{*}=Q(1 / n)$, then:

$$
\lim _{n \rightarrow \infty} P\left(B_{n}^{*} \leq x\right)=1-e^{-x^{\theta}},
$$

for $x>0$. The cdf of the $W$ distribution of parameters 1 and $\theta$ was obtained as limit; $\left\{B_{n}^{*}\right\}_{n}$ converged in distribution to a random variable with such a $W$ distribution.

Proof. We aimed to apply Theorem 8.3.2 of [34]. Based on the asymptotic of $T(t)$ in Theorem 1 , as $t \rightarrow \infty$, the following equivalence result held:

$$
T(t) \sim \frac{e^{2 \alpha \lambda t^{\theta}}}{\theta(2 \alpha \lambda)^{1 / \theta}} \Gamma\left(\frac{1}{\theta}, 2 \alpha \lambda t^{\theta}\right) \sim \frac{t^{1-\theta}}{2 \alpha \lambda} \frac{\Gamma\left(1 / \theta, 2 \alpha \lambda t^{\theta}\right)}{e^{-2 \alpha \lambda t^{\theta}}\left(2 \alpha \lambda t^{\theta}\right)^{1 / \theta-1}} \sim \frac{t^{1-\theta}}{2 \alpha \theta \lambda} .
$$

Hence, for $\theta=1$, we had:

$$
\lim _{t \rightarrow \infty} \frac{R(t+x T(t))}{R(t)}=\lim _{t \rightarrow \infty} \frac{e^{-2 \alpha \lambda\left(t+(2 \alpha \lambda)^{-1} x\right)}}{e^{-2 \alpha \lambda t}}=e^{-x} .
$$

The desired result immediately followed from Theorem 8.3.2 of [34]. Now, let us point out that, by virtue of Lemma 1 applied to the cdf of the ECSW distribution, we had:

$$
\lim _{t \rightarrow 0} \frac{F(t x)}{F(t)} \sim \lim _{t \rightarrow 0} \frac{1-e^{-\lambda(t x)^{\theta}}}{1-e^{-\lambda t^{\theta}}}=x^{\theta} .
$$

The proof of Theorem 2 became a direct application of Theorem 8.3.6 of [34].

Theorem 2 was useful for the study of the extreme value theory involving the ECSW distribution.

\subsubsection{The ECSP Distribution}

First of all, the ECSP distribution has the feature to have a bounded support, contrasting with the ECSW distribution. The cdf, pdf, and hrf of the ECSP distribution were obtain by considering the baseline distribution of the power $(\mathrm{P})$ distribution with sf and pdf defined as: $s(x ; \lambda, \theta)=1-(x / \lambda)^{\theta}$ and $g(x ; \lambda, \theta)=\theta \lambda^{-\theta} x^{\theta-1}$, respectively, where $0 \leq x \leq \lambda$ and $\lambda, \theta>0$. That is, for $0 \leq x \leq \lambda$, the cdf, pdf, and hrf of the ECSP distribution were:

$$
\begin{gathered}
F(x)=1-\left\{1-\cos \left[\frac{\pi}{2}\left(1-\left(\frac{x}{\lambda}\right)^{\theta}\right)\right]\right\}^{\alpha}, \\
f(x)=\frac{\alpha \theta \lambda^{-\theta} \pi}{2} x^{\theta-1} \sin \left[\frac{\pi}{2}\left(1-\left(\frac{x}{\lambda}\right)^{\theta}\right)\right]\left\{1-\cos \left[\frac{\pi}{2}\left(1-\left(\frac{x}{\lambda}\right)^{\theta}\right)\right]\right\}^{\alpha-1}
\end{gathered}
$$


and

$$
h(x)=\frac{\alpha \theta \lambda^{-\theta}(\pi / 2) x^{\theta-1} \sin \left[(\pi / 2)\left(1-(x / \lambda)^{\theta}\right)\right]}{1-\cos \left[(\pi / 2)\left(1-(x / \lambda)^{\theta}\right)\right]},
$$

respectively. These functions could be completed by the appropriate values for $x<0$ and $x>\lambda$.

Figure 2 shows plots of the pdf and hrf of the ECSP distribution. We saw that the pdf of the ECSP distribution was extremely flexible; increasing, decreasing, unimodal left-skewed, unimodal right-skewed, and bathtub shapes were observed. The hrf was mainly bathtub shaped. Thus, the ECSP distribution was ideal for modeling phenomena whose values were bounded and presented such features.
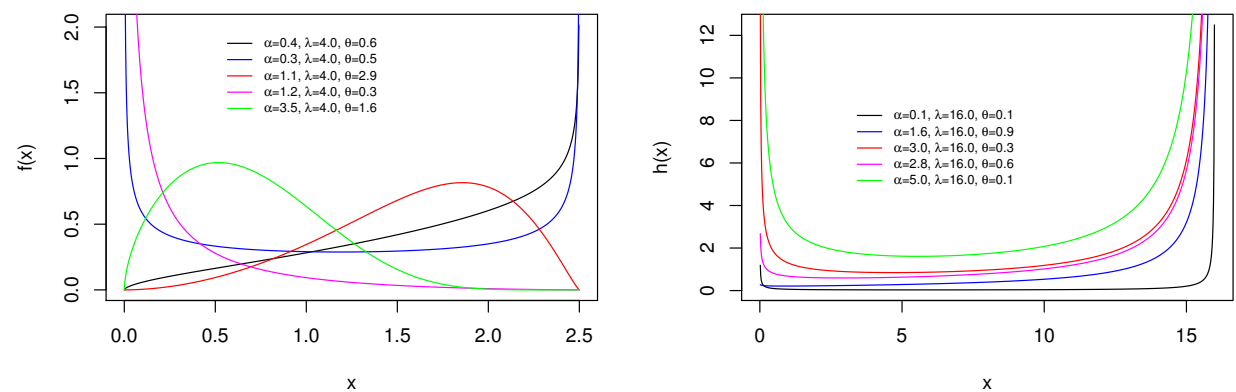

Figure 2. Plots of the pdf and hrf of the ECSP distribution for some parameter values.

A similar study as the one performed for the ECSW distribution could be conducted. Here, we chose to perform something different by focusing on the quantile analysis. Thus, the $\mathrm{qf}$ of the ECSP distribution was given as:

$$
Q(u)=\lambda\left(1-\frac{2}{\pi}\left[\arccos \left(1-(1-u)^{1 / \alpha}\right)\right]\right)^{1 / \theta}, \quad 0 \leq u \leq 1 .
$$

From this qf, we could define diverse quantile measures, such as Bowley's skewness and Moors' kurtosis of the ECSP distribution. Figure 3 displays the three-dimensional plots of these skewness and kurtosis measures. It was clear that both the skewness and kurtosis were increasing in $\alpha$ and decreasing in $\theta$, and were independent of $\lambda$.
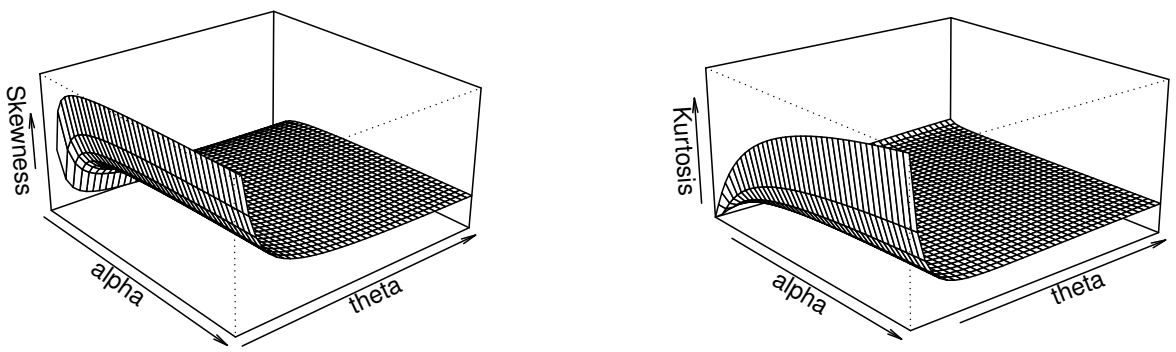

Figure 3. Plots of Bowley's skewness and Moors's kurtosis of the ECSP distribution.

\subsubsection{The ECSGHL Distribution}

The ECSGHL distribution could be viewed as a trigonometric extension of the generalized half-logistic (GHL) distribution introduced by [36]. It is a new three-parameter lifetime distribution such as the ECSW distribution. The cdf, pdf, and hrf of the ECSGHL distribution were obtain by considering the baseline distribution of the GHL distribution, with sf and pdf defined by: $s(x ; \lambda, \theta)=1-\left[\left(1-e^{-\lambda x}\right) /\left(1+e^{-\lambda x}\right)\right]^{\theta}$ and $g(x ; \lambda, \theta)=$ $\left[2 \lambda \theta e^{-\lambda x} /\left(1+e^{-\lambda x}\right)^{2}\right]\left[\left(1-e^{-\lambda x}\right) /\left(1+e^{-\lambda x}\right)\right]^{\theta-1}$, respectively, where $x, \lambda, \theta>0$. That is, for $x>0$, the cdf, pdf, and hrf of the ECSGHL distribution were: 


$$
\begin{gathered}
F(x)=1-\left\{1-\cos \left[\frac{\pi}{2}\left(1-\left(\frac{1-e^{-\lambda x}}{1+e^{-\lambda x}}\right)^{\theta}\right)\right]\right\}^{\alpha}, \\
f(x)=\frac{\alpha \lambda \theta \pi e^{-\lambda x}}{\left(1+e^{-\lambda x}\right)^{2}}\left(\frac{1-e^{-\lambda x}}{1+e^{-\lambda x}}\right)^{\theta-1} \sin \left[\frac{\pi}{2}\left(1-\left(\frac{1-e^{-\lambda x}}{1+e^{-\lambda x}}\right)^{\theta}\right)\right] \\
\times\left\{1-\cos \left[\frac{\pi}{2}\left(1-\left(\frac{1-e^{-\lambda x}}{1+e^{-\lambda x}}\right)^{\theta}\right)\right]\right\}^{\alpha-1}
\end{gathered}
$$

and

$$
\begin{aligned}
h(x)= & \frac{\alpha \theta \lambda \pi e^{-\lambda x}}{\left(1+e^{-\lambda x}\right)^{2}}\left(\frac{1-e^{-\lambda x}}{1+e^{-\lambda x}}\right)^{\theta-1} \times \\
& \frac{\sin \left[(\pi / 2)\left(1-\left[\left(1-e^{-\lambda x}\right) /\left(1+e^{-\lambda x}\right)\right]^{\theta}\right)\right]}{1-\cos \left[(\pi / 2)\left(1-\left[\left(1-e^{-\lambda x}\right) /\left(1+e^{-\lambda x}\right)\right]^{\theta}\right)\right]},
\end{aligned}
$$

respectively. Figure 4 shows plots of the pdf and hrf of the ECSGHL distribution. We observed that the pdf may have been decreasing, and was unimodal with right-skewed or almost symmetrical shapes. The platykurtic shape property was sometimes observed. The hrf had decreasing, increasing, and non-monotonic shapes. In light of the above remarks, the ECSGHL distribution was appropriate to model lifetime data with decreasing and right-skewed unimodal structures, with various symmetrical or platykurtic features.
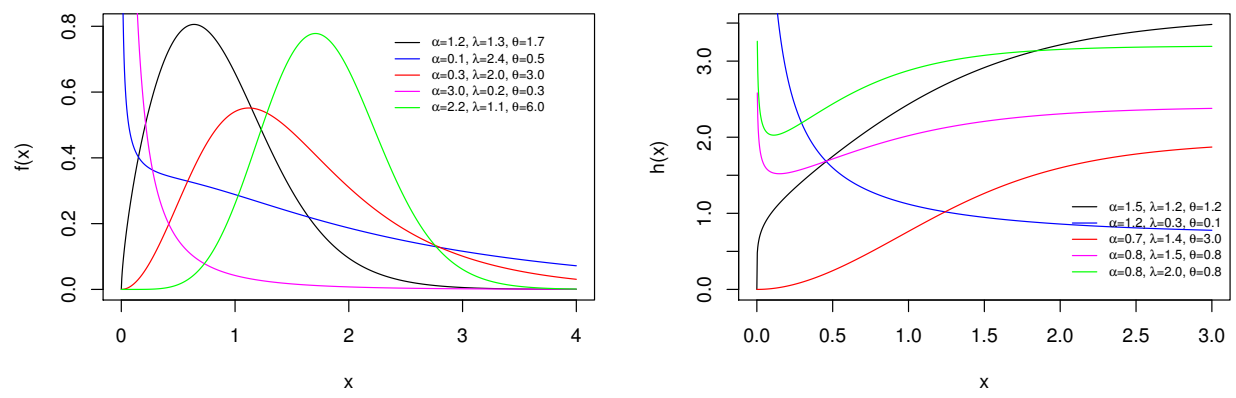

Figure 4. Plots of the pdf and hrf of the ECSGHL distribution for some parameter values.

Theoretical research comparable to that conducted for the ECSW and ECSP distributions could be carried out. For the sake of space, we concentrated our efforts on the statement of new results, with potential applications in the extreme value theory.

We began with a theorem on the asymptotic behavior of the mean residual life and mean reverse residual life.

Theorem 3. Let $X$ be a random variable following the ECSGHL distribution. Then,

- As $t \rightarrow \infty$, the mean residual life of $X$ satisfies:

$$
T(t) \sim \frac{1}{2 \alpha \lambda},
$$

- As $t \rightarrow 0$, the mean reverse residual life of $X$ satisfies:

$$
\bar{T}(t) \sim \frac{t}{\theta+1} .
$$


Proof. By applying Lemma 1 to the cdf of the ECSGHL distribution, as $x \rightarrow \infty$, we had:

$$
R(x) \sim \frac{\pi^{2 \alpha}}{8^{\alpha}}\left[1-\left(\frac{1-e^{-\lambda x}}{1+e^{-\lambda x}}\right)^{\theta}\right]^{2 \alpha} \sim 2^{\alpha}\left(\frac{\pi}{2}\right)^{2 \alpha} \theta^{2 \alpha} e^{-2 \alpha \lambda x} .
$$

This implies that, as $t \rightarrow \infty$,

$$
T(t)=\int_{0}^{\infty} \frac{R(x+t)}{R(t)} d x \sim e^{2 \alpha \lambda t} \int_{0}^{\infty} e^{-2 \alpha \lambda(x+t)} d x=\frac{1}{2 \alpha \lambda} .
$$

On the other hand, as $x \rightarrow 0$ from Lemma 1 adapted to the cdf of the ECSGHL distribution, we had:

$$
F(x) \sim \alpha \frac{\pi}{2}\left(\frac{1-e^{-\lambda x}}{1+e^{-\lambda x}}\right)^{\theta} \sim \alpha \frac{\pi \lambda^{\theta}}{2^{\theta+1}} x^{\theta} .
$$

Thus, as $t \rightarrow 0$, we had:

$$
\bar{T}(t)=\int_{0}^{t} \frac{F(x)}{F(t)} d x \sim t^{-\theta} \int_{0}^{t} x^{\theta} d x=\frac{t}{\theta+1} .
$$

Theorem 3 was proved.

The results in Theorem 3 could be used for several theoretical purposes. One of them on the extreme value theory was formulated in the next result.

Theorem 4. Let $\left\{X_{n}\right\}_{n}$ be a sequence of independent random variables with the ECSHL distribution.

- Let $B_{n}=\left(X_{n: n}-a_{n}\right) / b_{n}$ where $a_{n}=Q(1-1 / n)$ and $b_{n}=E\left[X-a_{n} \mid X>a_{n}\right]$, then:

$$
\lim _{n \rightarrow \infty} P\left(B_{n} \leq x\right)=e^{-e^{-x}},
$$

for $x \in \mathbb{R}$. The cdf of the standard Gumbel distribution was obtained as limit; $\left\{B_{n}\right\}_{n}$ converged in distribution to a random variable with such a Gumbel distribution.

- $\quad$ Let $B_{n}^{*}=\left(X_{1: n}-a_{n}^{*}\right) / b_{n}^{*}$ where $a_{n}^{*}=0$ and $b_{n}^{*}=Q(1 / n)$, then:

$$
\lim _{n \rightarrow \infty} P\left(B_{n}^{*} \leq x\right)=1-e^{-x^{\theta}},
$$

for $x>0$. The cdf of the $W$ distribution of parameters 1 and $\theta$ was obtained as limit; $\left\{B_{n}^{*}\right\}_{n}$ converged in distribution to a random variable with such a $W$ distribution.

Proof. The application of Theorem 8.3.2 of [34] was the key of the first point of proof. The asymptotic behavior of $T(t)$ studied in Theorem 3 gave:

$$
\lim _{t \rightarrow \infty} \frac{R(t+x T(t))}{R(t)} \sim \lim _{t \rightarrow \infty} \frac{e^{-2 \alpha \lambda(t+x /(2 \alpha \lambda))}}{e^{-2 \alpha \lambda t}}=e^{-x} .
$$

We obtained the desired result by applying Theorem 8.3.2 of [34].

On the other hand, notice that, thanks to Lemma 1 adapted to the cdf of the ECSGHL distribution, we had:

$$
\lim _{t \rightarrow 0} \frac{F(t x)}{F(t)} \sim \lim _{t \rightarrow 0} \frac{\alpha\left(\pi \lambda^{\theta} / 2^{\theta+1}\right)(t x)^{\theta}}{\alpha\left(\pi \lambda^{\theta} / 2^{\theta+1}\right) t^{\theta}}=x^{\theta} .
$$

A direct application of Theorem 8.3.6 of [34] gave the expected result. This ended the proof of Theorem 4. 


\section{Estimation}

In this section, we estimated the parameters of the ECSG family using maximum likelihood estimation (MLE), least-square estimation (LSE), percentile estimation (PE), and Bayes estimation (BE). Simulation studies were conducted using the ECSW distribution to examine the performance of these estimation techniques. Moreover, the expression of the stress-strength reliability parameter $R_{S}$, when parameter $\lambda$ was common, was obtained, and estimated by Bayes estimation (BE) under various loss functions.

\section{1. $M L E$}

Let $\left(X_{1}, \ldots, X_{n}\right)$ be a random sample of size $n$ from a distribution of the ECSG family, and $\left(x_{1}, \ldots, x_{n}\right)$ be an observation of this sample. Let $\Theta=(\alpha, \xi)^{T}$ be a vector of parameters; then, the maximum likelihood estimate of $\Theta$, denoted by $\hat{\Theta}=(\hat{\alpha}, \hat{\xi})^{T}$, was computed by the maximization of the log-likelihood function with respect to $\Theta$ given by:

$$
\begin{aligned}
L(\Theta) & =n \log \alpha+n \log \left(\frac{\pi}{2}\right)+\sum_{i=1}^{n} \log g\left(x_{i} ; \xi\right)+\sum_{i=1}^{n} \log \sin \left(\frac{\pi}{2} s\left(x_{i} ; \xi\right)\right) \\
& +(\alpha-1) \sum_{i=1}^{n} \log \left[1-\cos \left(\frac{\pi}{2} s\left(x_{i} ; \xi\right)\right)\right] .
\end{aligned}
$$

This maximization could be performed by solving the following nonlinear system of equations given by $\partial L(\Theta) / \partial \alpha=0$ and $\partial L(\Theta) / \partial \xi=0$. Here, we had:

$$
\frac{\partial L(\Theta)}{\partial \alpha}=\frac{n}{\alpha}+\sum_{i=1}^{n} \log \left[1-\cos \left(\frac{\pi}{2} s\left(x_{i} ; \xi\right)\right)\right]
$$

and

$$
\begin{aligned}
\frac{\partial L(\Theta)}{\partial \xi} & =\sum_{i=1}^{n} \frac{g^{\xi}\left(x_{i} ; \xi\right)}{g\left(x_{i} ; \xi\right)}+\frac{\pi}{2} \sum_{i=1}^{n} s^{\xi}\left(x_{i} ; \xi\right) \cot \left(\frac{\pi}{2} s\left(x_{i} ; \xi\right)\right) \\
& +(\alpha-1) \frac{\pi}{2} \sum_{i=1}^{n} \frac{s^{\xi}\left(x_{i} ; \xi\right) \sin \left((\pi / 2) s\left(x_{i} ; \xi\right)\right)}{1-\cos \left((\pi / 2) s\left(x_{i} ; \xi\right)\right)}
\end{aligned}
$$

where $g^{\xi}\left(x_{i} ; \xi\right)$ and $s^{\xi}\left(x_{i} ; \xi\right)$ indicate the partial derivative of $g\left(x_{i} ; \xi\right)$ and $s\left(x_{i} ; \xi\right)$, respectively, with respect to $\xi$. We gave the existence and uniqueness of the MLE of $\alpha$ : Let $U(\alpha ; \xi)$ be the right hand side of Equation (12). Then, from Equation (12), $\lim _{\alpha \rightarrow 0} U(\alpha ; \xi)=\infty$, and $\lim _{\alpha \rightarrow \infty} U(\alpha ; \xi)=\sum_{i=1}^{n} \log \left[1-\cos \left((\pi / 2) s\left(x_{i} ; \xi\right)\right)\right]<0$, thus, $U(\alpha ; \xi)$ is a decreasing function from positive to negative values. Since $\partial^{2} L(\Theta) / \partial \alpha^{2}=-n / \alpha^{2}<0, U(\alpha ; \xi)=0$ has a unique real root.

The observed Fisher information matrix was required for the interval estimation of the parameters. In the setting of the ECSG family, it was defined by:

$$
\hat{I}=-\left.\frac{\partial^{2} L(\Theta)}{\partial \Theta \partial \Theta^{T}}\right|_{\Theta=\hat{\Theta}} \text {. }
$$

The asymptotic distribution underlying $\hat{\Theta}$ could be approximated as the multivariate normal, $N_{k}\left(\Theta, \hat{I}^{-1}\right)$ under the usual condition for the parameters in the interior of the parameter space, but not on the boundary where $k$ is the total number of parameters. The elements of matrix $\hat{I}^{-1}$ can be requested from the authors.

For $r=1,2,3, \ldots, k$, let $\Theta_{r}$ be the $r^{\text {th }}$ component of $\Theta$. Then, a $100(1-\epsilon) \%$ asymptotic confidence interval for $\Theta_{r}$ was given by:

$$
A C I_{r}=\left(\hat{\Theta}_{r}-w_{\epsilon / 2} \sqrt{\hat{i}_{r r}}, \hat{\Theta}_{r}+w_{\epsilon / 2} \sqrt{\hat{i}_{r r}}\right),
$$


where $\hat{\Theta}_{r}$ is the $r^{\text {th }}$ component of $\hat{\Theta}, \hat{i}_{r r}$ is the $(r, r)^{\text {th }}$ diagonal element of $\hat{I}^{-1}$, and $w_{\epsilon / 2}$ is the quantile $1-\epsilon / 2$ of the standard normal distribution.

\section{2. $L S E$}

Here, we provided the LSE technique for the parameters of the ECSG family. Let $\left(X_{1: n}, \ldots, X_{n: n}\right)$ be an ordered version of $\left(X_{1}, \ldots, X_{n}\right)$, and $\left(x_{1: n}, \ldots, x_{n: n}\right)$ be an observation of this sample. Then, the least-square estimate for $\Theta=(\alpha, \bar{\xi})^{T}$, denoted by $\bar{\Theta}=(\bar{\alpha}, \bar{\xi})^{T}$, could be obtain by minimizing function $\ell(\Theta)$ with respect to $\Theta$ defined by:

$$
\ell(\Theta)=\sum_{i=1}^{n}\left\{1-\left[1-\cos \left(\frac{\pi}{2} s\left(x_{i: n} ; \xi\right)\right)\right]^{\alpha}-\frac{i}{n+1}\right\}^{2} .
$$

It was the solution of the following nonlinear equations, $\partial \ell(\Theta) / \partial \alpha=0$ and $\partial \ell(\Theta) / \partial \xi=$ 0 , where

$$
\frac{\partial \ell(\Theta)}{\partial \alpha}=-2 \sum_{i=1}^{n}\left\{1-\left[1-\cos \left(\frac{\pi}{2} s\left(x_{i: n} ; \xi\right)\right)\right]^{\alpha}-\frac{i}{n+1}\right\} u_{i}^{\alpha} \log \left(u_{i}\right)
$$

and

$$
\begin{gathered}
\frac{\partial \ell(\Theta)}{\partial \xi}=-\alpha \pi \sum_{i=1}^{n}\left\{1-\left[1-\cos \left(\frac{\pi}{2} s\left(x_{i: n} ; \xi\right)\right)\right]^{\alpha}-\frac{i}{n+1}\right\} \times \\
u_{i}^{\alpha-1} \sin \left(\frac{\pi}{2} s\left(x_{i: n} ; \xi\right)\right) s^{\xi}\left(x_{i: n} ; \xi\right),
\end{gathered}
$$

with $u_{i}=1-\cos \left((\pi / 2) s\left(x_{i: n} ; \xi\right)\right)$.

Based on Equation (3), the ECSG family could have a closed-form qf provided the baseline cdf had a closed-form qf. Hence, the unknown vector of parameters $\Theta$ could be estimated by equating the sample percentile points to the population percentile points. Let $u_{i}$ denote an estimate of $F\left(x_{i: n}\right)$; then, the percentile estimate of $\Theta$, denoted by $\tilde{\Theta}=(\tilde{\alpha}, \tilde{\xi})^{T}$, could be obtained by minimizing function $P(\Theta)$ with respect to $\Theta$ defined by:

$$
P(\Theta)=\sum_{i=1}^{n}\left\{x_{i: n}-q_{G}\left(1-\frac{2}{\pi}\left[\arccos \left(1-\left(1-u_{i}\right)^{1 / \alpha}\right)\right]\right)\right\}^{2}
$$

which also corresponds to the solutions of the following nonlinear equations: $\partial P(\Theta) / \partial \alpha=$ 0 and $\partial P(\Theta) / \partial \xi=0$.

\section{4. $B E$}

In this part, we proposed the use of the $\mathrm{BE}$ to estimate $\Theta$ under the square error loss function (SEL). The Bayes estimate of $\Theta$, denoted by $\hat{\Theta}$, was derived from the posterior distributions given the sample data. Let $N$ be the number of iterations and $K$ be the number of the burn-in samples; then, the SEL for the assumed prior distribution was $(\hat{\Theta}-\Theta)^{2}$ that minimized by the posterior mean as $\hat{\Theta}=[1 /(N-K)] \sum_{i=K+1}^{N} \hat{\Theta}^{(i)}$. The highest posterior pdf (HPD) credible interval for $\hat{\Theta}$ could be constructed using the technique derived by [37].

Let $\left(X_{1}, \ldots, X_{n}\right)$ be a random sample of size $n$ from a distribution of the ECSG family, and $\left(x_{1}, \ldots, x_{n}\right)$ be an observation of this sample. Assume that $\Theta$ is a random vector with components that are independent and follow a certain gamma distribution, e.g., $\alpha \sim \operatorname{Gamma}\left(a_{1}, b_{1}\right)=v_{1}(\alpha)$ and $\xi \sim \operatorname{Gamma}\left(a_{r}, b_{r}\right)=v_{r}(\xi), r=1, \ldots, m$, assuming that $\xi$ has $m$ parameters, i.e., gamma distributions are the prior distributions. Then, the likelihood function could be expressed as: 


$$
L(\Theta \mid \text { data })=\alpha^{n}\left(\frac{\pi}{2}\right)^{n} \prod_{i=1}^{n} g\left(x_{i} ; \xi\right) \prod_{i=1}^{n} \sin \left(\frac{\pi}{2} s\left(x_{i} ; \xi\right)\right) \prod_{i=1}^{n}\left(1-\cos \left[\frac{\pi}{2} s\left(x_{i} ; \xi\right)\right)\right]^{\alpha-1}
$$

The joint posterior pdf of $\Theta \mid$ data was given by:

$$
\pi(\Theta \mid \text { data })=\frac{L(\Theta \mid \text { data }) v_{1}(\alpha) v_{1}(\xi) \ldots v_{k}(\xi)}{\iiint \zeta(\text { data; } \Theta) d \alpha d \xi},
$$

where $\zeta($ data $; \Theta)=L(\Theta \mid$ data $) v_{1}(\alpha) \ldots v_{k}(\xi)$ is the join pdf for the data. We could obtain the marginal posterior pdf of $\alpha$ and $\xi_{r}$ for $r=1, \ldots, m$ from Equation (13) as:

$$
\pi_{1}(\alpha) \propto \alpha^{n+a_{1}-1} e^{-b_{1} \alpha} \prod_{i=1}^{n}\left[1-\cos \left(\frac{\pi}{2} s\left(x_{i} ; \xi\right)\right)\right]^{\alpha-1}
$$

and

$$
\pi_{r}(\xi) \propto \xi_{r}^{a_{r}-1} e^{-b_{r} \xi_{r}} \prod_{i=1}^{n} g\left(x_{i} ; \xi\right) \prod_{i=1}^{n} \sin \left(\frac{\pi}{2} s\left(x_{i} ; \xi\right)\right) \prod_{i=1}^{n}\left[1-\cos \left(\frac{\pi}{2} s\left(x_{i} ; \xi\right)\right)\right]^{\alpha-1} .
$$

It was clear that the related posterior distributions were not from well-known distributions. Consequently, we could use the Metropolis-Hastings algorithm with the normal distribution and the Gibbs sampling technique to generate samples from the posterior distributions. The algorithm was described step by step below (see [38-40]).

1. Start by initial guess $\left(\alpha^{(0)}, \xi^{(0)}\right)$;

2. Set $t=1$;

3. Use the Metropolis-Hastings algorithm to generate $\alpha^{(t)}$ from $\pi_{1}(\alpha)$;

4. Use the Metropolis-Hastings algorithm to generate $\xi^{(t)}$ from $\pi_{r}(\xi)$;

5. Set $t=t+1$;

6. Repeat steps three to five, $T$ times.

For a very large $T, \hat{\Theta}$ could be obtained based on the SEL, and the HPD credible interval could be constructed.

\subsection{Simulation Studies and Practice of the ECSW Model}

Simulation results from the ECSW model were collected to assess the MLE, LSE, PE, and $\mathrm{BE}$ of $\Theta$ under SEL. We proceed as follows: To begin, we generated 1000 samples of size $n \in\{50,60,70, \ldots, 300\}$ from the ECSW distribution for some parameter values. The bias and mean square error (MSE) of the estimates were computed using R3.5.3 [41]. In the case of $\mathrm{BE}$, we used $N=1000$ number of iterations and the first $10 \%$ as burn-in samples. We noticed that the estimation was good when all the hyperparameters were greater than one. The results of the simulation studies are illustrated in Figures 5-7. It was indicated that the MLE, SLE, PE, and BE showed consistency, and their MSE decreased as the sample size increased; the bias of some parameters was negative in some cases.
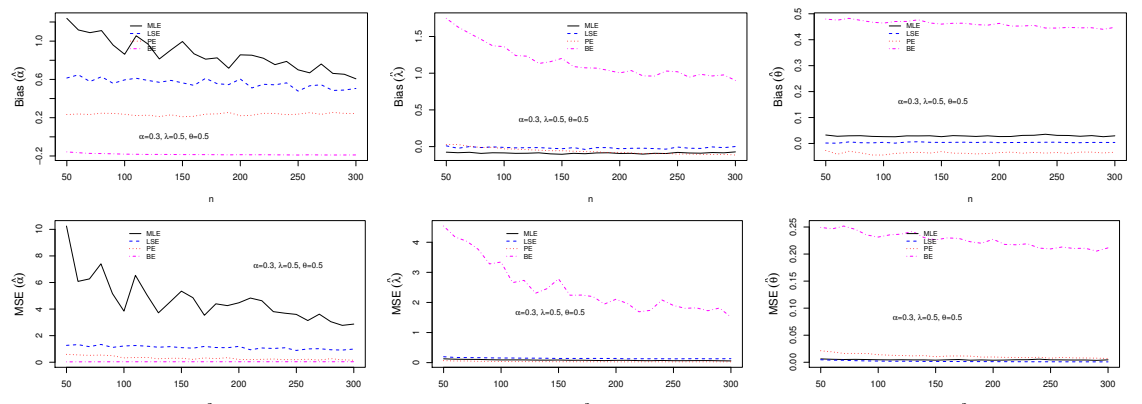

Figure 5. Plots of the bias and MSE for the different estimates of $\alpha=0.3, \lambda=0.5$ and $\theta=0.5$. 

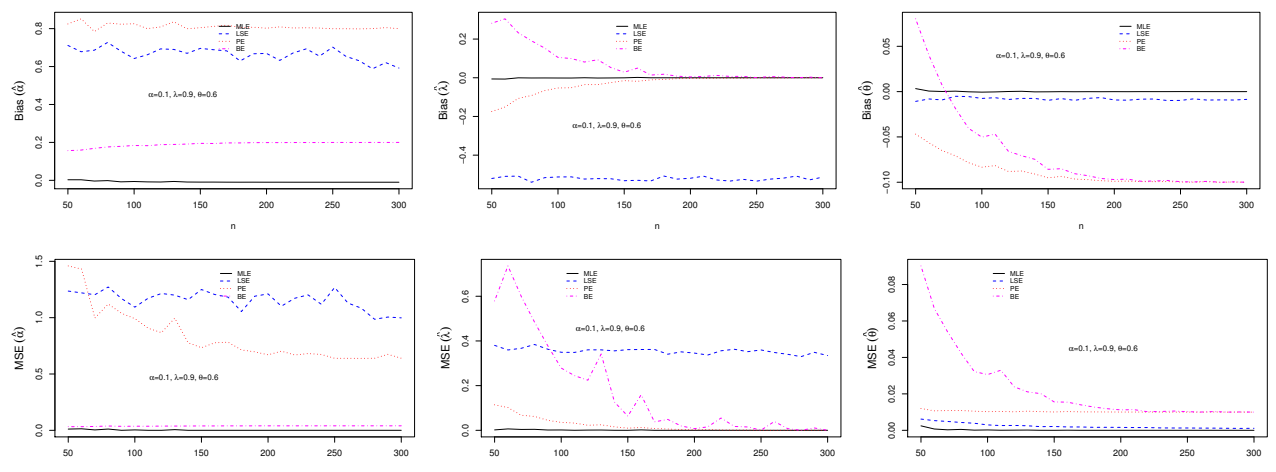

Figure 6. Plots of the bias and MSE for the different estimates of $\alpha=0.1, \lambda=0.9$ and $\theta=0.6$.
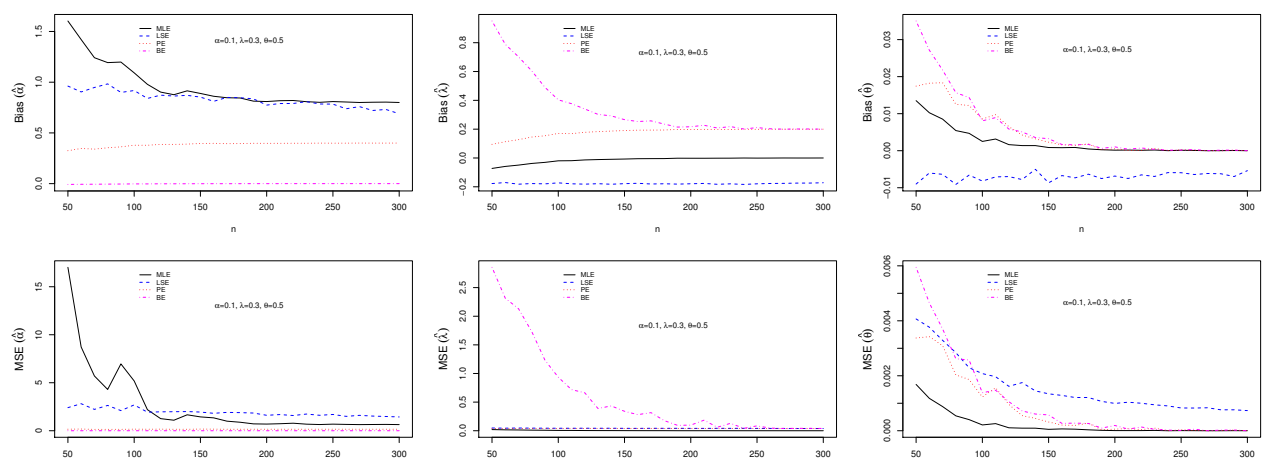

Figure 7. Plots of the bias and MSE for the different estimates of $\alpha=0.1, \lambda=0.3$ and $\theta=0.5$.

In practice, we fitted the ECSW model to real data using the MLE, LSE, PE, and BE. We compared the obtained fits using Kolmogorov-Smirnov (KS), Anderson-Darling (AD), and Cramér-von Mises (CVM), along with the $p$-values of their corresponding tests. The considered dataset represented 30 successive values for precipitation (in inches) in March for the Minneapolis/St. Paul area over 30 years, given in [42], and also studied by [43]. The data were: $0.77,1.74,0.81,1.20,1.95,1.20,0.47,1.43,3.37,2.20,3.00,3.09,1.51$, $2.10,0.52,1.62,1.31,0.32,0.59,0.81,2.81,1.87,1.18,1.35,4.75,2.48,0.96,1.89,0.90$, and 2.05.

The numerical results are given in Table 1 . It can be seen that the performance of the different estimation techniques gave a satisfactory result, with $p$-values almost equal to 1 . Therefore, each of them could be a good choice in the estimation of the parameters of the ECSG family in practice. Figure 8 shows the plots of the estimated pdfs and histogram, empirical cdf, and estimated cdfs. In particular, it was clear that the shape of the histogram was well captured by the estimated pdf curve. To complete this analysis, Figure 9 shows the iteration obtained from the BE. Figure 10 presents the posterior pdfs of each parameter from the BE.

Table 1. MLE, LSE, PE, BE, KS, AD, and CVM with their $p$-value in parenthesis of the precipitation data.

\begin{tabular}{lcccccc}
\hline ECSW & $\boldsymbol{\alpha}$ & $\boldsymbol{\lambda}$ & $\boldsymbol{\theta}$ & KS & AD & CVM \\
\hline MLE & 0.2182 & 0.8029 & 1.7340 & $0.0647(0.9987)$ & $0.1522(0.9545)$ & $0.0196(0.9690)$ \\
LSE & 0.1992 & 0.9374 & 1.6669 & $0.0598(0.9997)$ & $0.1429(0.9668)$ & $0.0184(0.9787)$ \\
PE & 0.2057 & 0.9962 & 1.4871 & $0.0838(0.9727)$ & $0.1232(0.9853)$ & $0.0157(0.9914)$ \\
BE & 1.8027 & 0.1192 & 1.7202 & $0.0729(0.9936)$ & $0.1889(0.8928)$ & $0.0253(0.9007)$ \\
\hline
\end{tabular}



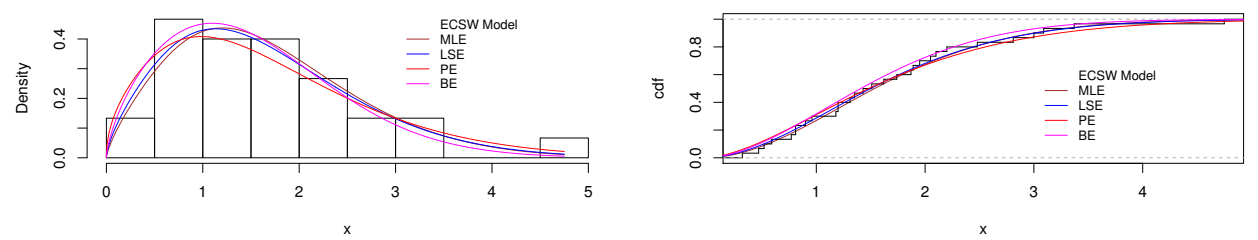

Figure 8. Plots of the estimated pdfs and histogram, empirical cdf and estimated cdfs for the precipitation data.
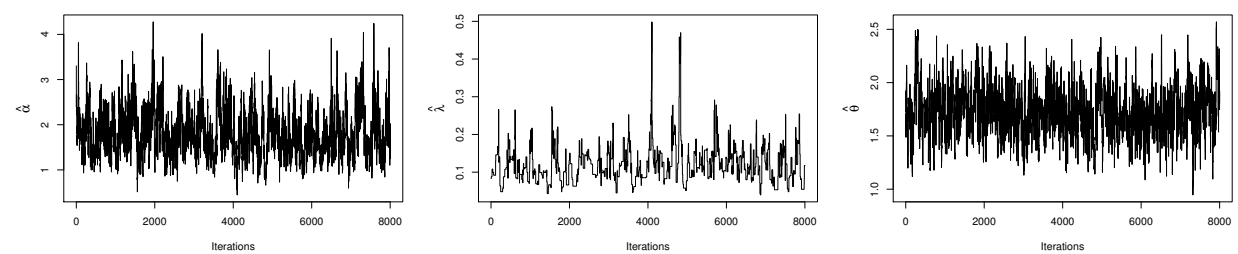

Figure 9. Plots of the iteration obtained from the BE for the precipitation data.
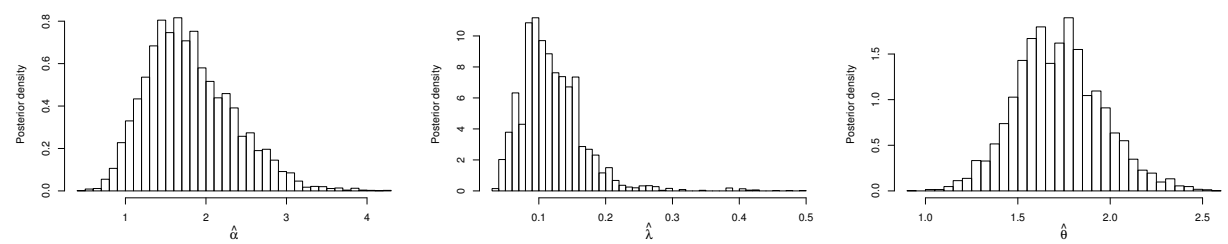

Figure 10. Plots of the posterior pdfs of each parameter from the BE for the precipitation data.

\subsection{BE of the Reliability Parameter of the ECSW Distribution}

Here, we obtained the expression of the stress-strength reliability parameter $R_{S}$ in the setting of the ECSW distribution when parameter $\lambda$ was common to both the stress and strength random variables. The parameter estimation was performed via the BE using gamma prior under various loss functions.

First, after a change of variable, we could express $R_{S}$ as:

$$
R_{s}=1-\alpha_{1} \int_{0}^{\frac{\pi}{2}} \sin \left(u_{1}\right)\left(1-\cos \left(u_{1}\right)\right)^{\alpha_{1}-1}\left(1-\cos \left(u_{2}\right)\right)^{\alpha_{2}} d u_{1}
$$

where $u_{2}=(\pi / 2) e^{-\lambda v_{u_{1}}}$ and $v_{u_{1}}=\left[-(1 / \lambda) \log \left(2 u_{1} / \pi\right)\right]^{\theta_{2} / \theta_{1}}$.

Let $\left(X_{1}, \ldots, X_{n}\right)$ be a random sample of size $n$ from the $\operatorname{ECSW}_{1}\left(\alpha_{1}, \theta_{1}, \lambda\right)$ distribution, and $\left(Y_{1}, \ldots, Y_{n}\right)$ be a random sample of size $m$ from the $\operatorname{ECSW}_{2}\left(\alpha_{2}, \theta_{2}, \lambda\right)$ distribution. Let $\left(x_{1}, \ldots, x_{n}\right)$ and $\left(y_{1}, \ldots, y_{n}\right)$ be observations of $\left(X_{1}, \ldots, X_{n}\right)$ and $\left(Y_{1}, \ldots, Y_{n}\right)$, respectively. Then, by using these observations, $R_{S}$ could easily be computed using mathematical software, such as R and MATLAB. We illustrated this claim via the BE of $R_{S}$ under the SEL, absolute error loss function (AEL), maximum a posteriori (MAP), general entropy loss function (GEL), and linear exponential loss function (LINEX).

Assume that $\Theta=\left(\alpha_{1}, \alpha_{2}, \theta_{1}, \theta_{2}, \lambda\right)$ is a random vector with independent components that are independent and follow a gamma distribution, meaning that gamma distributions are the prior distributions. More precisely, suppose that $\alpha_{1} \sim \operatorname{Gamma}\left(a_{1}, b_{1}\right)=v_{1}\left(\alpha_{1}\right)$, $\alpha_{2} \sim \operatorname{Gamma}\left(a_{2}, b_{2}\right)=v_{2}\left(\alpha_{2}\right), \theta_{1} \sim \operatorname{Gamma}\left(a_{3}, b_{3}\right)=v_{3}\left(\theta_{1}\right), \theta_{2} \sim \operatorname{Gamma}\left(a_{4}, b_{4}\right)=$ $v_{4}\left(\theta_{2}\right)$, and $\lambda \sim \operatorname{Gamma}\left(a_{5}, b_{5}\right)=v_{5}(\lambda)$. In this setting, the likelihood function was given by: 


$$
\begin{aligned}
L(\Theta \mid \text { data }) & =\alpha_{1}^{n} \lambda^{n+m} \theta_{1}^{n} \alpha_{2}^{m} \theta_{2}^{m}\left(\frac{\pi}{2}\right)^{n+m} \prod_{i=1}^{n} x_{i}^{\theta_{1}-1} \prod_{j=1}^{m} y_{j}^{\theta_{2}-1} e^{-\lambda \sum_{i=1}^{n} x_{i}^{\theta_{1}}} e^{-\lambda \sum_{j=1}^{m} y_{j}^{\theta_{2}}} \\
& \times \prod_{i=1}^{n} \sin \left(\frac{\pi}{2} e^{-\lambda x_{i}^{\theta_{1}}}\right) \prod_{j=1}^{m} \sin \left(\frac{\pi}{2} e^{-\lambda y_{j}^{\theta_{2}}}\right) \prod_{i=1}^{n}\left[1-\cos \left(\frac{\pi}{2} e^{-\lambda x_{i}^{\theta_{1}}}\right)\right]^{\alpha_{1}-1} \\
& \times \prod_{j=1}^{m}\left[1-\cos \left(\frac{\pi}{2} e^{-\lambda y_{j}^{\theta_{2}}}\right)\right]^{\alpha_{2}-1} .
\end{aligned}
$$

The joint posterior pdf of $\theta \mid$ data was given by:

$$
\pi(\Theta \mid \text { data })=\frac{L(\Theta \mid \text { data }) v_{1}\left(\alpha_{1}\right) v_{2}\left(\alpha_{2}\right) v_{3}\left(\theta_{2}\right) v_{4}\left(\theta_{2}\right) v_{5}(\lambda)}{\iiint \iint \zeta(\text { data } ; \Theta) d \alpha_{1} d \alpha_{2} d \theta_{1} d \theta_{2} d \lambda}
$$

where $\zeta($ data $; \Theta)=L(\Theta \mid$ data $) v_{1}\left(\alpha_{1}\right) v_{2}\left(\alpha_{2}\right) v_{3}\left(\theta_{2}\right) v_{4}\left(\theta_{2}\right) v_{5}(\lambda)$ is the join pdf for the data. The marginal posterior pdf of $\Theta$ could be obtained from Equation (15) as:

$$
\begin{gathered}
\pi_{1}\left(\alpha_{1}\right) \propto \alpha_{1}^{n+a_{1}-1} e^{-b_{1} \alpha_{1}} \prod_{i=1}^{n}\left[1-\cos \left(\frac{\pi}{2} e^{-\lambda x_{i}^{\theta_{1}}}\right)\right]^{\alpha_{1}-1}, \\
\pi_{2}\left(\alpha_{2}\right) \propto \alpha_{2}^{n+a_{2}-1} e^{-b_{2} \alpha_{2}} \prod_{j=1}^{m}\left[1-\cos \left(\frac{\pi}{2} e^{-\lambda y_{j}^{\theta_{2}}}\right)\right]^{\alpha_{2}-1}, \\
\pi_{3}\left(\theta_{1}\right) \propto \theta_{1}^{n+a_{3}-1} \prod_{i=1}^{n} x_{i}^{\theta_{1}-1} e^{-\lambda \sum_{i=1}^{m} x_{i}^{\theta_{1}}-b_{3} \theta_{1}} \prod_{i=1}^{n} \sin \left(\frac{\pi}{2} e^{-\lambda x_{i}^{\theta_{1}}}\right) \\
\times \prod_{i=1}^{n}\left[1-\cos \left(\frac{\pi}{2} e^{-\lambda x_{i}^{\theta_{1}}}\right)\right]^{\alpha_{1}-1}, \\
\pi_{4}\left(\theta_{2}\right) \propto \theta_{2}^{n+a_{4}-1} \prod_{j=1}^{m} y_{j}^{\theta_{2}-1} e^{-\lambda \sum_{j=1}^{m} y_{j}^{\theta_{2}}-b_{4} \theta_{2}} \prod_{j=1}^{m} \sin \left(\frac{\pi}{2} e^{-\lambda y_{j}^{\theta_{2}}}\right) \\
\times \prod_{j=1}^{m}\left[1-\cos \left(\frac{\pi}{2} e^{-\lambda y_{j}^{\theta_{2}}}\right)\right]^{\alpha_{2}-1}
\end{gathered}
$$

and

$$
\begin{aligned}
\pi_{5}(\lambda) & \propto \lambda^{n+m+a_{5}-1} e^{-b_{5} \lambda-\lambda\left(\sum_{i=1}^{n} x_{i}^{\theta_{1}}-\sum_{j=1}^{m} y_{j}^{\theta_{1}}\right)} \prod_{i=1}^{n} \sin \left(\frac{\pi}{2} e^{-\lambda x_{i}^{\theta_{1}}}\right) \\
& \times \prod_{j=1}^{m} \sin \left(\frac{\pi}{2} e^{-\lambda y_{j}^{\theta_{2}}}\right) \prod_{i=1}^{n}\left[1-\cos \left(\frac{\pi}{2} e^{-\lambda x_{i}^{\theta_{1}}}\right)\right]^{\alpha_{1}-1} \\
& \times \prod_{j=1}^{m}\left[1-\cos \left(\frac{\pi}{2} e^{-\lambda y_{j}^{\theta_{2}}}\right)\right]^{\alpha_{2}-1} .
\end{aligned}
$$

The posterior distributions were not from any recognized distributions. Therefore, we employed the use of the Metropolis-Hasting algorithm with the normal distribution and the Gibbs sampling technique to generate samples from the posterior distributions. The step-by-step algorithm could be deducted from Section 3.4.

The estimate of $R_{S}=R_{S}(\Theta)$ as defined in Equation (14) was $\hat{R}_{S}=R_{\mathcal{S}}(\hat{\Theta})$, where $\hat{\Theta}=\left(\hat{\alpha}_{1}, \hat{\alpha}_{2}, \hat{\theta}_{1}, \hat{\theta}_{2}, \hat{\lambda}\right)^{T}$ was derived from the BE. Here, $\hat{\Theta}$ was computed from the posterior distributions given the sample data. Let $N$ be the number of iterations and $M$ be the burn-in. The estimate that minimized SEL for the assumed prior distribution was the 
estimates mean defined by $\hat{R}_{s, S E L}=[1 /(N-M)] \sum_{i=M+1}^{N} \hat{R}_{S}^{(i)}$; the AEL was minimized by the posterior median as $\hat{R}_{S, A E L}$; the MAP depended on the likelihood function and prior distribution, $\hat{R}_{S, M A P}$ was defined by the value that maximized the posterior distribution; the GEL was minimized by $\hat{R}_{s, G E L}=\left\{[1 /(N-M)] \sum_{i=M+1}^{N}\left(\hat{R}_{s}^{(i)}\right)^{-c}\right\}^{-1 / c}$; the LINEX was minimized by estimate $\hat{R}_{s, L I N}=(-1 / c) \log \left\{[1 /(N-M)] \sum_{i=M+1}^{N} e^{c \hat{R}_{s}^{(i)}}\right\}$. In addition, the HPD credible interval for $R_{S}$ could be constructed as discussed in [37].

\subsection{Simulation Studies for the Reliability Parameter from the ECSW Distribution}

In this part, simulation studies were conducted to examine the Bayes estimates of $R_{s}$. A sample size of 1000 was generated for various sample sizes $n$ and $m$ from the $\operatorname{ECSW}_{1}\left(\alpha_{1}, \theta_{1}, \lambda\right)$ and $\operatorname{ECSW}_{2}\left(\alpha_{2}, \theta_{2}, \lambda\right)$ distributions, respectively, for some different parameter values. We considered various cases of $(n, m)$ as $(20,20),(30,20),(30,40),(50,40)$, and $(60,60)$. The Bayes estimates of $R_{S}$ under various loss functions were computed, and the bias, MSE, 95\% credible interval, and coverage probability (CP) were obtained. We considered a number of 1000 iterations, and the first $20 \%$ as the burn-in; in a similar way, the estimation was satisfactory when all the hyperparameters selected were greater than one. The results of the simulation are given in Table 2.

We saw that, as the two sample sizes increased, the MSE decreased; the estimated value of $R_{S}$ went to its actual value, and the average length of the confidence interval (ALCI) decreased as the sample size increased. Furthermore, the CP went to the nominal level of $95 \%$.

Table 2. Actual parameter values and $R_{S}$; Bayes estimates of $R_{S}$ under various loss functions; MSE with bias in parenthesis, ALCI with CP in parenthesis of the estimates.

\begin{tabular}{|c|c|c|c|c|c|c|c|c|c|c|c|c|}
\hline$\left(\alpha_{1}, \alpha_{2}, \theta_{1}, \theta_{2}, \lambda, R_{s}\right)$ & $(n, m)$ & $\hat{R}_{s, S E L}$ & $M S E_{S E L}$ & $\hat{R}_{s, A E L}$ & $M S E_{A E L}$ & $\hat{R}_{s, M A P}$ & $M S E_{M A P}$ & $\hat{R}_{s, G E L}$ & $M S E_{G E L}$ & $\hat{R}_{s, L I N}$ & $M S E_{L I N}$ & ALCI \\
\hline \multirow[t]{5}{*}{$(1.0,1.0,1.0,1.0,1.0,0.5000)$} & $(20,20)$ & 0.4995 & $\begin{array}{c}0.0059 \\
(-0.0005)\end{array}$ & 0.5008 & $\begin{array}{c}0.0061 \\
(0.0008)\end{array}$ & 0.5028 & $\begin{array}{c}0.0071 \\
(0.0023)\end{array}$ & 0.4751 & $\begin{array}{c}0.0072 \\
(-0.0249)\end{array}$ & 0.4925 & $\begin{array}{c}0.0060 \\
(-0.0075)\end{array}$ & $\begin{array}{c}0.3186 \\
(0.96)\end{array}$ \\
\hline & $(30,20)$ & 0.4613 & $\begin{array}{c}0.0057 \\
(0.0082)\end{array}$ & 0.4619 & $\begin{array}{c}0.0054 \\
(0.0088)\end{array}$ & 0.4624 & $\begin{array}{c}0.0066 \\
(0.0094)\end{array}$ & 0.4394 & $\begin{array}{c}0.0058 \\
(-0.0137)\end{array}$ & 0.4554 & $\begin{array}{c}0.0051 \\
(0.0024)\end{array}$ & $\begin{array}{l}0.2907 \\
(0.95)\end{array}$ \\
\hline & $(30,40)$ & 0.5017 & $\begin{array}{c}0.0035 \\
(0.0017)\end{array}$ & 0.5024 & $\begin{array}{c}0.0036 \\
(0.0024)\end{array}$ & 0.5029 & $\begin{array}{c}0.0044 \\
(0.0029)\end{array}$ & 0.4876 & $\begin{array}{c}0.0039 \\
(-0.0124)\end{array}$ & 0.4974 & $\begin{array}{c}0.0035 \\
(-0.0026)\end{array}$ & $\begin{array}{l}0.2511 \\
(0.96)\end{array}$ \\
\hline & $(50,40)$ & 0.4998 & $\begin{array}{c}0.0029 \\
(-0.0002)\end{array}$ & 0.5003 & $\begin{array}{c}0.0030 \\
(0.0003)\end{array}$ & 0.5011 & $\begin{array}{c}0.0034 \\
(0.0011)\end{array}$ & 0.4884 & $\begin{array}{c}0.0032 \\
(-0.0116)\end{array}$ & 0.4962 & $\begin{array}{c}0.0029 \\
(-0.0037)\end{array}$ & $\begin{array}{c}0.2272 \\
(0.96)\end{array}$ \\
\hline & $(60,60)$ & 0.5018 & $\begin{array}{c}0.0024 \\
(0.0018)\end{array}$ & 0.5021 & $\begin{array}{c}0.0025 \\
(0.0021) \\
\end{array}$ & 0.5027 & $\begin{array}{c}0.0029 \\
(0.0027) \\
\end{array}$ & 0.4935 & $\begin{array}{c}0.0026 \\
(-0.0066)\end{array}$ & 0.4991 & $\begin{array}{c}0.0024 \\
(-0.0009)\end{array}$ & $\begin{array}{c}0.1964 \\
(0.95) \\
\end{array}$ \\
\hline \multirow[t]{5}{*}{$(2.0,3.5,0.9,0.6,2.0,0.8396)$} & $(20,20)$ & 0.8302 & $\begin{array}{c}0.0034 \\
(-0.0094)\end{array}$ & 0.8371 & $\begin{array}{c}0.0034 \\
(-0.0025)\end{array}$ & 0.8493 & $\begin{array}{c}0.0039 \\
(0.0097)\end{array}$ & 0.8226 & $\begin{array}{c}0.0040 \\
(-0.0017)\end{array}$ & 0.8264 & $\begin{array}{c}0.0037 \\
(-0.0132)\end{array}$ & $\begin{array}{l}.2213 \\
(0.93)\end{array}$ \\
\hline & $(30,20)$ & 0.8289 & $\begin{array}{c}0.0030 \\
(-0.0107)\end{array}$ & 0.8348 & $\begin{array}{c}0.0030 \\
(-0.0048)\end{array}$ & 0.8443 & $\begin{array}{c}0.0034 \\
(0.0047)\end{array}$ & 0.8225 & $\begin{array}{c}0.0035 \\
(-0.0171)\end{array}$ & 0.8256 & $\begin{array}{c}0.0032 \\
(-0.0139)\end{array}$ & $\begin{array}{c}0.2060 \\
(0.94)\end{array}$ \\
\hline & $(30,40)$ & 0.8385 & $\begin{array}{c}0.0022 \\
(-0.0011)\end{array}$ & 0.8428 & $\begin{array}{c}0.0023 \\
(0.0033)\end{array}$ & 0.8493 & $\begin{array}{c}0.0026 \\
(0.0097)\end{array}$ & 0.8344 & $\begin{array}{c}0.0024 \\
(-0.0052)\end{array}$ & 0.8363 & $\begin{array}{c}0.0023 \\
(-0.0032)\end{array}$ & $\begin{array}{c}0.1698 \\
(0.91)\end{array}$ \\
\hline & $(50,40)$ & 0.8359 & $\begin{array}{c}0.0017 \\
(-0.0037)\end{array}$ & 0.8392 & $\begin{array}{c}0.0017 \\
(-0.0003)\end{array}$ & 0.8452 & $\begin{array}{c}0.0019 \\
(0.0056)\end{array}$ & 0.8326 & $\begin{array}{c}0.0018 \\
(-0.0069)\end{array}$ & 0.8341 & $\begin{array}{c}0.0018 \\
(-0.0054)\end{array}$ & $\begin{array}{c}0.1526 \\
(0.93)\end{array}$ \\
\hline & $(60,60)$ & 0.8404 & $\begin{array}{c}0.0012 \\
(0.0008)\end{array}$ & 0.8429 & $\begin{array}{c}0.0012 \\
(0.0034)\end{array}$ & 0.8469 & $\begin{array}{c}0.0015 \\
(0.0074) \\
\end{array}$ & 0.8381 & $\begin{array}{c}0.0013 \\
(-0.0015) \\
\end{array}$ & 0.8392 & $\begin{array}{c}0.0012 \\
(-0.0004) \\
\end{array}$ & $\begin{array}{r}0.1307 \\
(0.93) \\
\end{array}$ \\
\hline \multirow[t]{5}{*}{$(1.3,1.4,0.5,0.6,0.5,0.4995)$} & $(20,20)$ & 0.4970 & $\begin{array}{c}0.0063 \\
(-0.0025)\end{array}$ & 0.4985 & $\begin{array}{c}0.0066 \\
(-0.0009)\end{array}$ & 0.5009 & $\begin{array}{c}0.0080 \\
(0.0014)\end{array}$ & 0.4704 & $\begin{array}{c}0.0078 \\
(-0.0291)\end{array}$ & 0.4891 & $\begin{array}{c}0.0064 \\
(-0.0099)\end{array}$ & $\begin{array}{c}0.3261 \\
(0.96)\end{array}$ \\
\hline & $(30,20)$ & 0.5005 & $\begin{array}{c}0.0059 \\
(0.0010)\end{array}$ & 0.5016 & $\begin{array}{c}0.0062 \\
(0.0021)\end{array}$ & 0.5030 & $\begin{array}{c}0.0074 \\
(0.0036)\end{array}$ & 0.4779 & $\begin{array}{c}0.0068 \\
(-0.0216)\end{array}$ & 0.4940 & $\begin{array}{c}0.0059 \\
(-0.0055)\end{array}$ & $\begin{array}{c}0.3039 \\
(0.95)\end{array}$ \\
\hline & $(30,40)$ & 0.4962 & $\begin{array}{c}0.0044 \\
(-0.0032)\end{array}$ & 0.4971 & $\begin{array}{c}0.0045 \\
(-0.0024)\end{array}$ & 0.4978 & $\begin{array}{c}0.0054 \\
(-0.0016)\end{array}$ & 0.4807 & $\begin{array}{c}0.0049 \\
(-0.0188)\end{array}$ & 0.4916 & $\begin{array}{c}0.0044 \\
(-0.0079)\end{array}$ & $\begin{array}{l}0.2585 \\
(0.95)\end{array}$ \\
\hline & $(50,40)$ & 0.4959 & $\begin{array}{c}0.0030 \\
(-0.0035)\end{array}$ & 0.4964 & $\begin{array}{c}0.0031 \\
(-0.0031)\end{array}$ & 0.4967 & $\begin{array}{c}0.0038 \\
(-0.0027)\end{array}$ & 0.4838 & $\begin{array}{c}0.0034 \\
(-0.0156)\end{array}$ & 0.4922 & $\begin{array}{c}0.0031 \\
(-0.0072)\end{array}$ & $\begin{array}{l}0.2317 \\
(0.96)\end{array}$ \\
\hline & $(60,60)$ & 0.4974 & $\begin{array}{c}0.0024 \\
(-0.0020) \\
\end{array}$ & 0.4978 & $\begin{array}{c}0.0024 \\
(-0.0017) \\
\end{array}$ & 0.4986 & $\begin{array}{c}0.0029 \\
(-0.0009) \\
\end{array}$ & 0.4887 & $\begin{array}{c}0.0026 \\
(-0.0108) \\
\end{array}$ & 0.4947 & $\begin{array}{c}0.0024 \\
(-0.0048) \\
\end{array}$ & $\begin{array}{c}0.1995 \\
(0.96) \\
\end{array}$ \\
\hline \multirow[t]{5}{*}{$(1.3,1.2,1.5,1.5,1.5,0.4800)$} & $(20,20)$ & 0.4873 & $\begin{array}{c}0.0064 \\
(0.0073)\end{array}$ & 0.4883 & $\begin{array}{c}0.0067 \\
(0.0084)\end{array}$ & 0.4890 & $\begin{array}{c}0.0080 \\
(0.0099)\end{array}$ & 0.4609 & $\begin{array}{c}0.0077 \\
(-0.0190)\end{array}$ & 0.4800 & $\begin{array}{c}0.0064 \\
(-0.0001)\end{array}$ & $\begin{array}{l}0.3265 \\
(0.95)\end{array}$ \\
\hline & $(30,20)$ & 0.4850 & $\begin{array}{c}0.0062 \\
(0.0050)\end{array}$ & 0.4856 & $\begin{array}{c}0.0064 \\
(0.0056)\end{array}$ & 0.4859 & $\begin{array}{c}0.0075 \\
(0.0059)\end{array}$ & 0.4626 & $\begin{array}{c}0.0072 \\
(-0.0174)\end{array}$ & 0.4786 & $\begin{array}{c}0.0062 \\
(-0.0014)\end{array}$ & $\begin{array}{c}0.3038 \\
(0.93)\end{array}$ \\
\hline & $(30,40)$ & 0.4841 & $\begin{array}{c}0.0042 \\
(0.0041)\end{array}$ & 0.4846 & $\begin{array}{c}0.0044 \\
(0.0045)\end{array}$ & 0.4849 & $\begin{array}{c}0.0053 \\
(0.0049)\end{array}$ & 0.4687 & $\begin{array}{c}0.0047 \\
(-0.0113)\end{array}$ & 0.4796 & $\begin{array}{c}0.0043 \\
(-0.0004)\end{array}$ & $\begin{array}{c}0.2574 \\
(0.94)\end{array}$ \\
\hline & $(50,40)$ & 0.4824 & $\begin{array}{c}0.0032 \\
(0.0024)\end{array}$ & 0.4827 & $\begin{array}{c}0.0033 \\
(0.0027)\end{array}$ & 0.4832 & $\begin{array}{c}0.0038 \\
(0.0032)\end{array}$ & 0.4701 & $\begin{array}{c}0.0035 \\
(-0.0099)\end{array}$ & 0.4787 & $\begin{array}{c}0.0032 \\
(-0.0013)\end{array}$ & $\begin{array}{c}0.2319 \\
(0.96)\end{array}$ \\
\hline & $(60,60)$ & 0.4810 & $\begin{array}{c}0.0024 \\
(0.0010)\end{array}$ & 0.4813 & $\begin{array}{c}0.0025 \\
(0.0013)\end{array}$ & 0.4809 & $\begin{array}{c}0.0029 \\
(0.0009)\end{array}$ & 0.4720 & $\begin{array}{c}0.0026 \\
(-0.0080)\end{array}$ & 0.4783 & $\begin{array}{c}0.0025 \\
(-0.0017)\end{array}$ & $\begin{array}{c}0.2005 \\
(0.95)\end{array}$ \\
\hline
\end{tabular}


Table 2. Cont.

\begin{tabular}{|c|c|c|c|c|c|c|c|c|c|c|c|c|}
\hline$\left(\alpha_{1}, \alpha_{2}, \theta_{1}, \theta_{2}, \lambda, R_{s}\right)$ & $(n, m)$ & $\hat{R}_{s, S E L}$ & $M S E_{S E L}$ & $\hat{R}_{s, A E L}$ & $M S E_{A E L}$ & $\hat{R}_{S, M A P}$ & $M S E_{M A P}$ & $\hat{R}_{s, G E L}$ & $M S E_{G E L}$ & $\hat{R}_{s, L I N}$ & $M S E_{L I N}$ & ALCI \\
\hline \multirow[t]{5}{*}{$(2.0,3.0,1.0,1.0,1.0,0.6000)$} & $(20,20)$ & 0.5924 & $\begin{array}{c}0.0077 \\
(-0.0076)\end{array}$ & 0.5953 & $\begin{array}{c}0.0080 \\
(-0.0047)\end{array}$ & 0.6000 & $\begin{array}{c}0.0095 \\
(0.0001)\end{array}$ & 0.5711 & $\begin{array}{c}0.0094 \\
(-0.0289)\end{array}$ & 0.5851 & $\begin{array}{c}0.0080 \\
(-0.0149)\end{array}$ & $\begin{array}{c}0.3231 \\
(0.93)\end{array}$ \\
\hline & $(30,20)$ & 0.5941 & $\begin{array}{c}0.0063 \\
(-0.0059)\end{array}$ & 0.5966 & $\begin{array}{c}0.0065 \\
(-0.0034)\end{array}$ & 0.6016 & $\begin{array}{c}0.0076 \\
(0.0016)\end{array}$ & 0.5766 & $\begin{array}{c}0.0075 \\
(-0.0234)\end{array}$ & 0.5880 & $\begin{array}{c}0.0065 \\
(-0.0120)\end{array}$ & $\begin{array}{c}0.2974 \\
(0.93)\end{array}$ \\
\hline & $(30,40)$ & 0.5955 & $\begin{array}{c}0.0043 \\
(-0.0045)\end{array}$ & 0.5975 & $\begin{array}{c}0.0044 \\
(-0.0025)\end{array}$ & 0.6009 & $\begin{array}{c}0.0050 \\
(0.0009)\end{array}$ & 0.5830 & $\begin{array}{c}0.0049 \\
(-0.0169)\end{array}$ & 0.5909 & $\begin{array}{c}0.0044 \\
(-0.0091)\end{array}$ & $\begin{array}{c}0.2557 \\
(0.94)\end{array}$ \\
\hline & $(50,40)$ & 0.5970 & $\begin{array}{c}0.0033 \\
(-0.0030)\end{array}$ & 0.5986 & $\begin{array}{c}0.0034 \\
(-0.0014)\end{array}$ & 0.6006 & $\begin{array}{c}0.0039 \\
(0.0006)\end{array}$ & 0.5875 & $\begin{array}{c}0.0037 \\
(-0.0125)\end{array}$ & 0.5935 & $\begin{array}{c}0.0034 \\
(-0.0065)\end{array}$ & $\begin{array}{c}0.2268 \\
(0.95)\end{array}$ \\
\hline & $(60,60)$ & 0.5948 & $\begin{array}{c}0.0028 \\
(-0.0052)\end{array}$ & 0.5960 & $\begin{array}{c}0.0027 \\
(-0.0040)\end{array}$ & 0.5975 & $\begin{array}{c}0.0032 \\
(-0.0025)\end{array}$ & 0.5877 & $\begin{array}{c}0.0029 \\
(-0.0123)\end{array}$ & 0.5921 & $\begin{array}{c}0.0027 \\
(-0.0079)\end{array}$ & $\begin{array}{l}0.1977 \\
(00.94)\end{array}$ \\
\hline \multirow[t]{5}{*}{$(0.9,0.8,0.6,0.7,0.8,0.4531)$} & $(20,20)$ & 0.4633 & $\begin{array}{c}0.0059 \\
(0.0102)\end{array}$ & 0.4641 & $\begin{array}{c}0.0062 \\
(0.0110)\end{array}$ & 0.4661 & $\begin{array}{c}0.0078 \\
(0.0131)\end{array}$ & 0.4362 & $\begin{array}{c}0.0066 \\
(-0.0169)\end{array}$ & 0.4562 & $\begin{array}{c}0.0056 \\
(0.0031)\end{array}$ & $\begin{array}{c}0.3195 \\
(0.96)\end{array}$ \\
\hline & $(30,20)$ & 0.4565 & $\begin{array}{c}0.0045 \\
(0.0035)\end{array}$ & 0.4569 & $\begin{array}{c}0.0047 \\
(0.0039)\end{array}$ & 0.4568 & $\begin{array}{c}0.0057 \\
(0.0037)\end{array}$ & 0.4330 & $\begin{array}{c}0.0053 \\
(-0.0201)\end{array}$ & 0.4504 & $\begin{array}{c}0.0045 \\
(-0.0027)\end{array}$ & $\begin{array}{r}0.2987 \\
(0.96)\end{array}$ \\
\hline & $(30,40)$ & 0.4617 & $\begin{array}{c}0.0036 \\
(0.0087)\end{array}$ & 0.4622 & $\begin{array}{c}0.0038 \\
(0.0091)\end{array}$ & 0.4619 & $\begin{array}{c}0.0045 \\
(0.0089)\end{array}$ & 0.4459 & $\begin{array}{c}0.0039 \\
(-0.0072)\end{array}$ & 0.4573 & $\begin{array}{c}0.0036 \\
(0.0043)\end{array}$ & $\begin{array}{c}0.2531 \\
(0.96)\end{array}$ \\
\hline & $(50,40)$ & 0.4542 & $\begin{array}{c}0.0027 \\
(0.0012)\end{array}$ & 0.4541 & $\begin{array}{c}0.0028 \\
(0.0011)\end{array}$ & 0.4543 & $\begin{array}{c}0.0032 \\
(0.0012)\end{array}$ & 0.4416 & $\begin{array}{c}0.0030 \\
(-0.0114)\end{array}$ & 0.4507 & $\begin{array}{c}0.0027 \\
(-0.0024)\end{array}$ & $\begin{array}{c}0.2267 \\
(0.97)\end{array}$ \\
\hline & $(60,60)$ & 0.4579 & $\begin{array}{c}0.0022 \\
(0.0049) \\
\end{array}$ & 0.4580 & $\begin{array}{c}0.0022 \\
(0.0049) \\
\end{array}$ & 0.4579 & $\begin{array}{c}0.0027 \\
(0.0048) \\
\end{array}$ & 0.4488 & $\begin{array}{c}0.0023 \\
(-0.0042) \\
\end{array}$ & 0.4553 & $\begin{array}{c}0.0022 \\
(0.0022) \\
\end{array}$ & $\begin{array}{c}0.1952 \\
(0.96) \\
\end{array}$ \\
\hline \multirow[t]{5}{*}{$(1.1,1.5,1.2,1.3,1.0,0.5551)$} & $(20,20)$ & 0.5380 & $\begin{array}{c}0.0067 \\
(-0.0161)\end{array}$ & 0.5412 & $\begin{array}{c}0.0068 \\
(-0.0139)\end{array}$ & 0.5447 & $\begin{array}{c}0.0079 \\
(-0.0105)\end{array}$ & 0.5146 & $\begin{array}{c}0.0089 \\
(-0.0405)\end{array}$ & 0.5315 & $\begin{array}{c}0.0070 \\
(-0.0237)\end{array}$ & $\begin{array}{l}0.3285 \\
(0.95)\end{array}$ \\
\hline & $(30,20)$ & 0.5463 & $\begin{array}{c}0.0060 \\
(-0.0089)\end{array}$ & 0.5482 & $\begin{array}{c}0.0062 \\
(-0.0069)\end{array}$ & 0.5513 & $\begin{array}{c}0.0071 \\
(-0.0039)\end{array}$ & 0.5264 & $\begin{array}{c}0.0074 \\
(-0.0289)\end{array}$ & 0.5399 & $\begin{array}{c}0.0062 \\
(-0.0153)\end{array}$ & $\begin{array}{c}0.3037 \\
(0.94)\end{array}$ \\
\hline & $(30,40)$ & 0.5422 & $\begin{array}{c}0.0044 \\
(-0.0129)\end{array}$ & 0.5435 & $\begin{array}{c}0.0045 \\
(-0.0116)\end{array}$ & 0.5455 & $\begin{array}{c}0.0053 \\
(-0.0097)\end{array}$ & 0.5281 & $\begin{array}{c}0.0053 \\
(-0.0269)\end{array}$ & 0.5376 & $\begin{array}{c}0.0046 \\
(-0.0176)\end{array}$ & $\begin{array}{c}0.2598 \\
(0.94)\end{array}$ \\
\hline & $(50,40)$ & 0.5477 & $\begin{array}{c}0.0032 \\
(-0.0074)\end{array}$ & 0.5488 & $\begin{array}{c}0.0033 \\
(-0.0063)\end{array}$ & 0.5501 & $\begin{array}{c}0.0038 \\
(-0.0050)\end{array}$ & 0.5369 & $\begin{array}{c}0.0037 \\
(-0.0183)\end{array}$ & 0.5441 & $\begin{array}{c}0.0033 \\
(-0.0111)\end{array}$ & $\begin{array}{c}0.2311 \\
(0.95)\end{array}$ \\
\hline & $(60,60)$ & 0.5478 & $\begin{array}{c}0.0026 \\
(-0.0072)\end{array}$ & 0.5487 & $\begin{array}{c}0.0026 \\
(-0.0064)\end{array}$ & 0.5496 & $\begin{array}{c}0.0030 \\
(-0.0054)\end{array}$ & 0.5399 & $\begin{array}{c}0.0029 \\
(-0.0152)\end{array}$ & 0.5451 & $\begin{array}{c}0.0026 \\
(-0.0100)\end{array}$ & $\begin{array}{c}0.2004 \\
(0.96)\end{array}$ \\
\hline
\end{tabular}

\section{Real Data Illustration}

We provided an illustrative performance of the ECSG family through the ECSP, ECSGHL, and ECSW models using real datasets. The performance of the ECSP and ECSGHL models were compared with some other existing models, while the ECSW model was demonstrated as a good candidate in stress-strength parameter estimation in practice. The model parameters were estimated by the MLE. We compared the fit of the models by the Akaike information criterion (AIC), consistent Akaike information criterion (CAIC), Bayesian information criterion (BIC), KS, AD, and CVM, along with the $p$-values for the related test when possible. The model with the smallest value of AIC, BIC, KS, AD, and CVM, and the greatest value of $p$-values, fit the data better than the other. The competing models included the generalized modified Weibull-Poisson (GMWP) model, generalized modified Weibull-Geometric (GMWG) model, generalized modified Weibull-logarithmic (GMWL) model by [44], Poisson-odd generalized uniform (POGE-U) model by [45], exponentiated generalized linear exponential (EGLE) model by [46], gamma-uniform (GU) model by [47], generalized linear failure rate (GLFR) model by [48], beta Weibull (BW) model by [49], generalized modified Weibull (GMW) model by [50], modified Weibull distribution (MW) model by [51], generalized linear exponential (GLE) model by [52], beta-modified Weibull (BMW) model by [53], power (P) model, exponentiated generalized standardized half-logistic (EGSHL) model by [54], Poisson half-logistic (PHL) model by [55], Olapade half-logistic (OHL) model by [56], beta half-logistic (BHL) model by [57], half-logistic Poisson (HLP) model by [58], generalized half-logistic (GHL) model by [59], and complementary Poisson generalized half-logistic (CPGHL) model [60].

\subsection{Real Data I}

We fitted the ECSP model to the given data and compared it with some popular models, including those used previously in the study of that set. The dataset provided by [61] contained the lifetimes of fifty devices. They were given by: 21, 32, 36, 40, 45, 46, 47, $50,55,60,63,63,67,67,67,67,72,75,83,84,84,84,85,85,85,85,85,86,86,0.1,0.2,1,1,79$, $82,82,1,1,1,2,3,6,7,11,12,18,18,18,18$, and 18 .

The total time on the test (TTT) is presented in Figure 11. This figure indicated that the data had an underlying hrf with a bathtub shape. This case was covered by the hrf of the ECSP model. Table 3 gives the numerical results obtained. It is shown that the ECSP model provided a better fit than the other considered models. Figure 12 illustrates the histogram with the estimated pdf, and empirical cdf with the estimated cdf of the ECSP 
model. Figure 13 shows the plots of the empirical and estimated hrf, and the empirical and estimated chrf of the ECSP model. Figure 14 shows the plots of the profile-log likelihood functions to illustrate the uniqueness of the estimates, and the quantile-quantile (QQ) plot to check the adequacy of the estimated ECSP model.

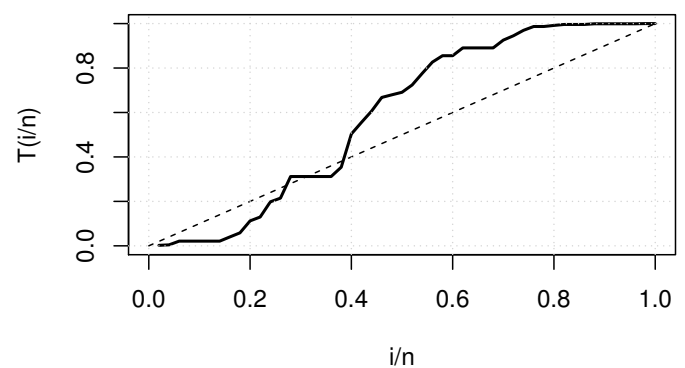

Figure 11. TTT plots for the lifetimes for real data I.

Table 3. Maximum likelihood estimates, L, AIC, CAIC, BIC, KS ( $p$-value) for real data I.

\begin{tabular}{|c|c|c|c|c|c|c|c|c|c|c|c|c|}
\hline Distributions & $\hat{\alpha}$ & $\hat{\beta}$ & $\hat{\lambda}$ & $\hat{a}$ & $\hat{b}$ & $\hat{\gamma}$ & $\hat{\theta}$ & $L$ & AIC & CAIC & BIC & KS ( $p$-Value) \\
\hline ECSP & 0.2118 & - & 86.0100 & - & - & - & 0.3551 & -202.59 & 411.19 & 411.71 & 416.92 & $0.0821(0.862)$ \\
\hline GU & 0.267 & 51.942 & - & 0.09 & 86.713 & - & - & -207.33 & 418.65 & 414.83 & 426.30 & $0.1520(0.198)$ \\
\hline $\mathrm{TUq}$ & - & - & -0.1900 & 0.0999 & 86.0000 & 0.9262 & - & -212.86 & 433.72 & 434.61 & 441.37 & $0.1212(0.421)$ \\
\hline BMW & $2.4 \times 10^{-4}$ & 0.054 & - & 0.197 & 0.165 & 1.380 & - & -220.28 & 450.56 & 440.78 & 460.12 & $0.1303(0.364)$ \\
\hline BW & $1.0 \times 10^{-5}$ & - & - & 0.129 & 0.070 & 3.320 & - & -223.11 & 454.22 & 446.40 & 461.87 & $0.1246(0.419)$ \\
\hline MW & 0.062 & 0.023 & - & - & - & 0.355 & - & -226.16 & 460.31 & 452.44 & 466.05 & $0.1338(0.332)$ \\
\hline EGLE & - & - & - & $3.3 \times 10^{-3}$ & $1.7 \times 10^{-4}$ & 4.564 & 0.112 & -224.34 & 456.67 & 448.85 & 464.32 & $0.1475(0.206)$ \\
\hline GLE & - & - & - & $9.6 \times 10^{-3}$ & $4.5 \times 10^{-4}$ & 0.730 & - & -235.93 & 477.85 & 471.98 & 483.59 & $0.1598(0.139)$ \\
\hline GLFR & - & - & - & $3.8 \times 10^{-3}$ & $3.1 \times 10^{-4}$ & - & 0.533 & -233.15 & 472.29 & 466.42 & 478.03 & $0.1620(0.129)$ \\
\hline POGE-U & 0.022 & 0.371 & 1.769 & - & 87.010 & - & - & -206.68 & 419.34 & 413.47 & 425.08 & $0.0923(0.754)$ \\
\hline GMWP & $5.4 \times 10^{-8}$ & 0.134 & 0.084 & - & - & 2.137 & 0.638 & -220.88 & 451.75 & 441.98 & 461.31 & $0.1400(0.281)$ \\
\hline GMWL & 2.130 & 2.682 & 0.013 & - & - & 0.278 & 1.000 & -217.77 & 445.53 & 435.76 & 455.09 & $0.1311(0.357)$ \\
\hline GMWG & $9.4 \times 10^{-8}$ & 0.123 & 0.075 & - & - & 2.228 & 0.464 & -220.78 & 451.55 & 441.78 & 461.11 & $0.1346(0.326)$ \\
\hline GMW & $1.0 \times 10^{-5}$ & 0.065 & - & 0.223 & - & 1.371 & - & -221.40 & 452.81 & 442.99 & 460.46 & $0.1464(0.234)$ \\
\hline $\mathrm{P}$ & - & - & 86.0100 & - & - & - & 0.7270 & -219.89 & 433.78 & 444.03 & 447.60 & $0.9999\left(8.9 \times 10^{-16}\right)$ \\
\hline
\end{tabular}
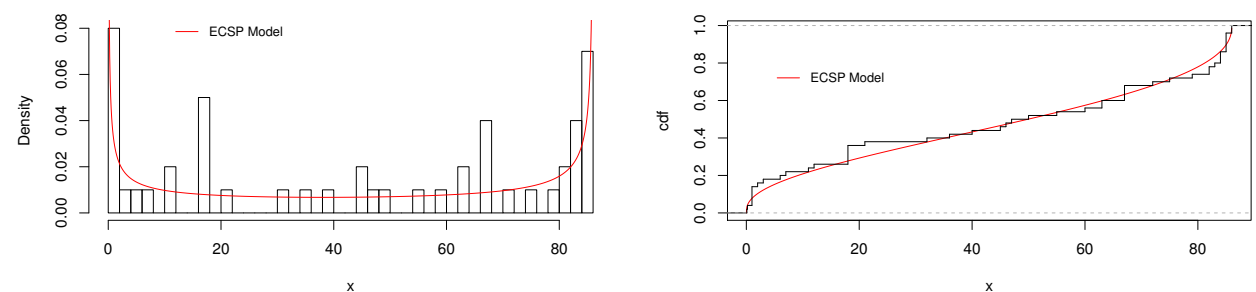

Figure 12. Plots of the histogram with the estimated pdf, and empirical cdf with estimated cdf of the ECSP model for real data I.
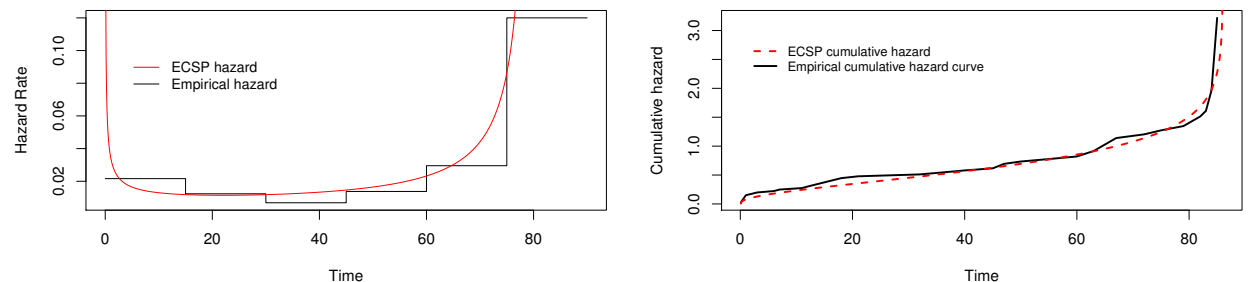

Figure 13. Plots of the empirical and estimated hrf, and the empirical and estimated chrf of the ECSP model for real data I. 

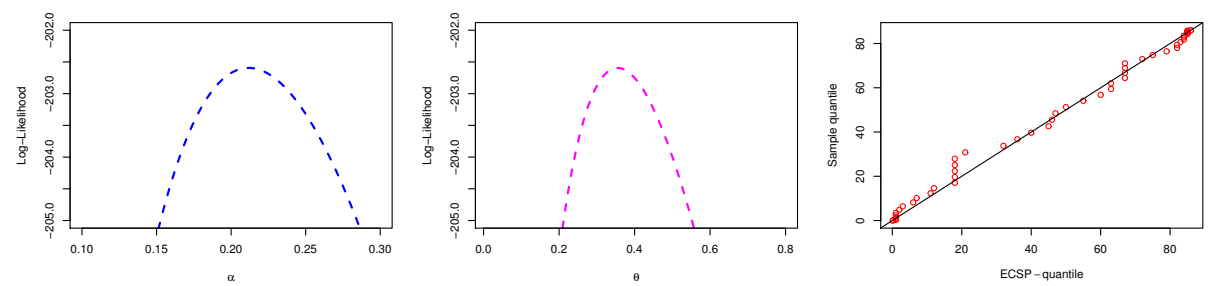

Figure 14. Plots of the profile-log likelihood functions, and the quantile-quantile plot of the ECSP model for real data I.

\subsection{Real Data II}

The ECSGHL model was considered to fit the dataset provided by [62]. The dataset consisted of the 100 observations on the breaking stress of carbon fibers (in Gba). The data were: $2.76,4.91,3.68,1.84,1.59,3.19,1.57,0.81,5.56,1.73,1.59,2,1.22,1.12,1.71,2.17,1.17$, $5.08,2.48,1.18,3.51,2.17,1.69,1.25,4.38,1.84,0.39,3.68,2.48,0.85,1.61,2.79,4.7,2.03,1.8$, $1.57,1.08,2.03,1.61,2.12,1.89,2.88,2.82,2.05,3.65,3.7,2.74,2.73,2.5,3.6,3.11,3.27,2.87$, $1.47,3.11,4.42,2.41,3.19,3.22,1.69,3.28,3.09,1.87,3.15,4.9,3.75,2.43,2.95,2.97,3.39,2.96$, $2.53,2.67,2.93,3.22,3.39,2.81,4.2,3.33,2.55,3.31,3.31,2.85,2.56,3.56,3.15,2.35,2.55,2.59$, $2.38,2.81,2.77,2.17,2.83,1.92,1.41,3.68,2.97,1.36$, and 0.98 . The fit of the ECSGHL model was compared with some existing models using some of the model selection criteria and goodness-of-fit tests mentioned above.

Figure 15 displays the TTT plot, showing that the data possessed an underlying increasing hrf. The ECSGHL model was able to treat this case. The numerical results are given in Table 4. From this table, it was clear that the ECSGHL model provided a better fit than the other competing models. Figure 16 shows the plots of the histogram with the estimated pdf, and the empirical cdf with the estimated cdf of the ECSGHL model. Figure 17 shows the plots of the empirical and estimated hrf, the empirical and estimated chrf model, and the QQ plot of the ECSGHL model, while Figure 18 shows the plots of the profile-log likelihood functions of the fitted ECSGHL model.

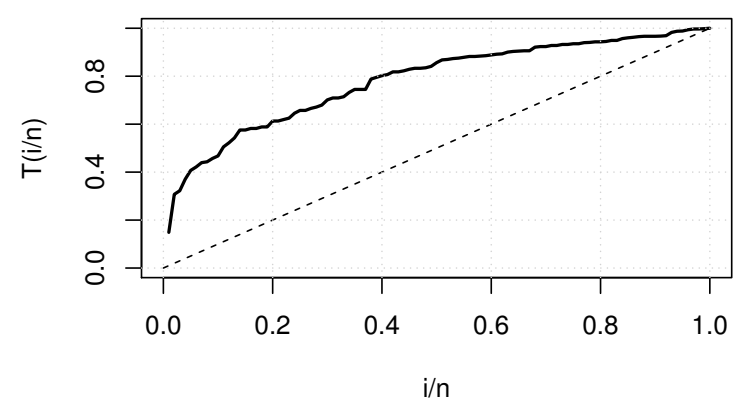

Figure 15. TTT plot for real data II.

Table 4. Maximum likelihood estimates, $\mathrm{L}, \mathrm{AIC}, \mathrm{CAIC}, \mathrm{BIC}, \mathrm{KS}, \mathrm{AD}, \mathrm{CVM}$ with $p$-value in parenthesis for real data II.

\begin{tabular}{lccccccccccccc}
\hline Distributions & $\hat{\alpha}$ & $\hat{\beta}$ & $\hat{\lambda}$ & $\hat{a}$ & $\hat{b}$ & $\hat{\theta}$ & $L$ & AIC & CAIC & BIC & KS & AD & CVM \\
\hline ECSGHL & 2942.23 & - & 0.0033 & - & - & 2.7980 & -141.42 & 289.04 & 283.11 & 296.86 & $0.0604(0.860)$ & $0.4118(0.334)$ & $0.0619(0.353)$ \\
GHL & 1.0795 & - & - & - & - & 4.9029 & -144.73 & 293.45 & 293.58 & 298.66 & $0.0977(0.295)$ & $0.9859(0.013)$ & $0.1923(0.007)$ \\
PHL & 1.2004 & - & 7.2596 & - & - & - & -143.12 & 290.24 & 290.36 & 295.45 & $0.0895(0.399)$ & $0.7502(0.049)$ & $0.1492(0.024)$ \\
EGSHL & - & - & - & 1.1133 & 5.0189 & - & -144.55 & 293.10 & 293.22 & 298.31 & $0.1011(0.258)$ & $0.9685(0.014)$ & $0.1891(0.007)$ \\
CPGHL & 1.2860 & - & 2.9110 & - & - & 3.1660 & -142.45 & 290.90 & 291.15 & 298.71 & $0.0827(0.501)$ & $0.6271(0.099)$ & $0.1248(0.051)$ \\
BHL & 0.1328 & - & - & 4.6873 & 6.7492 & - & -142.20 & 290.41 & 290.66 & 298.22 & $0.0849(0.467)$ & $0.5847(0.125)$ & $0.1141(0.071)$ \\
HLP & 0.1250 & - & 6.0979 & - & - & - & -197.92 & 399.85 & 399.97 & 405.06 & $0.3202(2.5 \mathrm{e}-9)$ & $0.8115(0.034)$ & $0.1588(0.018)$ \\
OHL & 0.7987 & - & - & - & - & 0.6459 & -180.92 & 365.85 & 365.97 & 371.06 & $0.2778(3.9 \mathrm{e}-7)$ & $0.6329(0.096)$ & $0.1247(0.051)$ \\
\hline
\end{tabular}



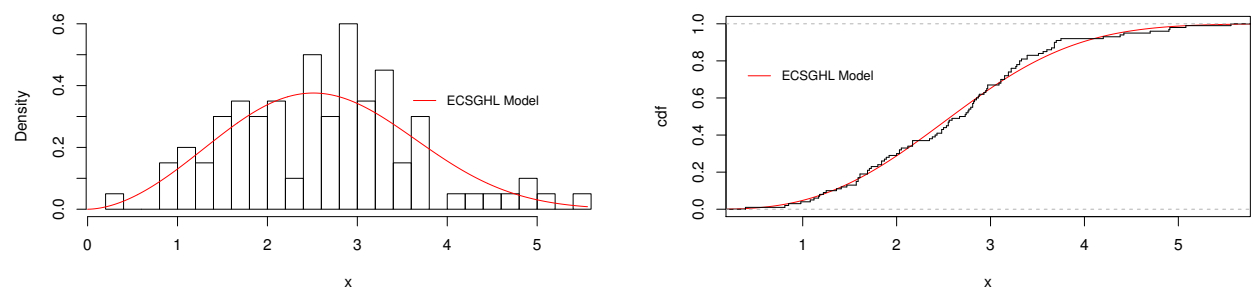

Figure 16. Plots of the histogram with the estimated pdf, and the empirical cdf with the estimated cdf of the ECSGHL model for real data II.
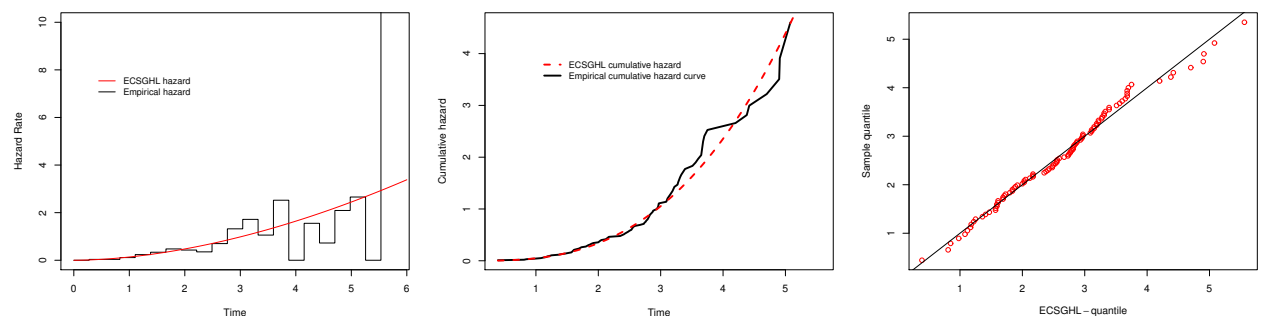

Figure 17. Plots of the empirical and estimated hrf, the empirical and estimated chrf, and QQ plot of the ECSGHL model for real data II.
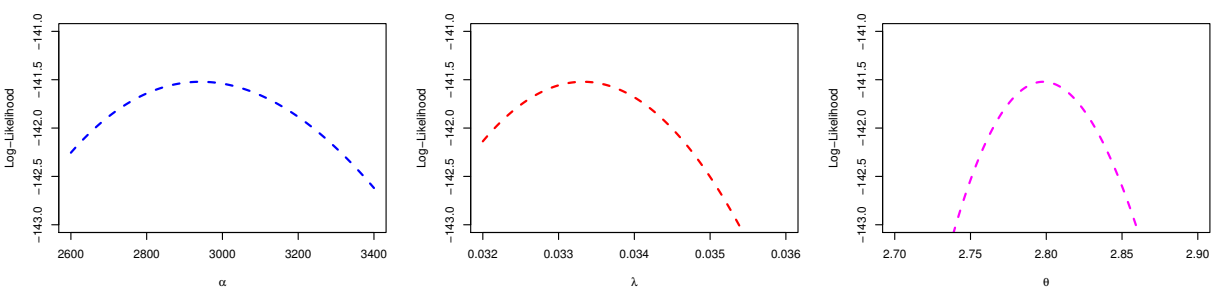

Figure 18. Plots of the profile-log likelihood functions of the fitted ECSGHL model for real data II.

\subsection{Real Data III}

Here, the performance of the ECSW model in reliability studies was demonstrated using a real dataset. The stress-strength reliability parameter $R_{s}$, when parameter $\lambda$ was common, was estimated using the BE using gamma prior distributions under the SEL, AEL, MAP, GEL, and LINEX. The HPD credible interval of $R_{S}$ was obtained for the GEL and LINEX (with the choice $c=2$ ). We considered 1000 iterations and the first $20 \%$ as the burn-in. The dataset was the strength data measured in GPA, for single carbon fibers and impregnated 1000-carbon fiber tows provided by [63]. Single fibers were tested under tension at gauge lengths of 1,10,20, and $50 \mathrm{~mm}$. Impregnated tows of 1000 fibers were tested at gauge lengths of 20,50,150, and $300 \mathrm{~mm}$. We considered the single fibers of $20 \mathrm{~mm}$ as (first data, $n=69$ ), and $10 \mathrm{~mm}$ (second data, $m=63$ ) in gauge lengths. These datasets were also studied by [64]. The data were given below:

First data: $0.312,0.314,0.479,0.552,0.700,0.803,0.861,0.865,0.944,0.958,0.966,0.997$, $1.006,1.021,1.027,1.055,1.063,1.098,1.140,1.179,1.224,1.240,1.253,1.270,1.272,1.274$, $1.301,1.301,1.359,1.382,1.382,1.426,1.434,1.435,1.478,1.490,1.511,1.514,1.535,1.554$, $1.566,1.570,1.586,1.629,1.633,1.642,1.648,1.684,1.697,1.726,1.770,1.773,1.800,1.809$, $1.818,1.821,1.848,1.880,1.954,2.012,2.067,2.084,2.090,2.096,2.128,2.233,2.433,2.585$, and 2.585 .

Second data: $0.101,0.332,0.403,0.428,0.457,0.550,0.561,0.596,0.597,0.645,0.654$, $0.674,0.718,0.722,0.725,0.732,0.775,0.814,0.816,0.818,0.824,0.859,0.875,0.938,0.940$, $1.056,1.117,1.128,1.137,1.137,1.177,1.196,1.230,1.325,1.339,1.345,1.420,1.423,1.435$, $1.443,1.464,1.472,1.494,1.532,1.546,1.577,1.608,1.635,1.693,1.701,1.737,1.754,1.762$, $1.828,2.052,2.071,2.086,2.171,2.224,2.227,2.425,2.595$, and 3.220.

The KS measure was used to show how the ECSW model provided a good fit to the two datasets. The numerical results for the Bayes estimates of the parameters under 
the SEL were: $\hat{\alpha}_{1}=0.7594, \hat{\alpha}_{2}=1.4136, \hat{\theta}_{1}=2.8335, \hat{\theta}_{2}=2.0313$, and $\hat{\lambda}=0.1921$. The KS value for the first dataset was $K S_{1}=0.0675$ ( $p$-value $\left.=0.8901\right)$, while for the second dataset was $K S_{2}=0.0787(p$-value $=0.8016)$. Figure 19 shows the $c d f$ of the first and second datasets with the fitted $\mathrm{ECSW}_{1}$ and $\mathrm{ECSW}_{2}$ models, respectively, indicating how well the ECSW model fit the two datasets by the proposed BE. The estimated values of the reliability parameter under various loss functions were $\hat{R}_{s, S E L}=0.5897, \hat{R}_{s, A E L}=0.5890$, $\hat{R}_{s, M A P}=0.5859, \hat{R}_{s, G E L}=0.5844$, and $\hat{R}_{s, L I N E X}=0.5877$, with a confidence interval of $(0.5006,0.6794)$. It was clear that the estimated values of $R_{S}$ under the various loss functions were very close, and the confidence interval was good. Thus, the ECSW model could be considered as a good candidate for stress-strength reliability studies. Figure 20 shows the plots of the histogram and estimated values of $R_{S}$ obtained from the Metropolis-Hastings algorithm and the Gibbs sampling technique of the Bayes estimates.
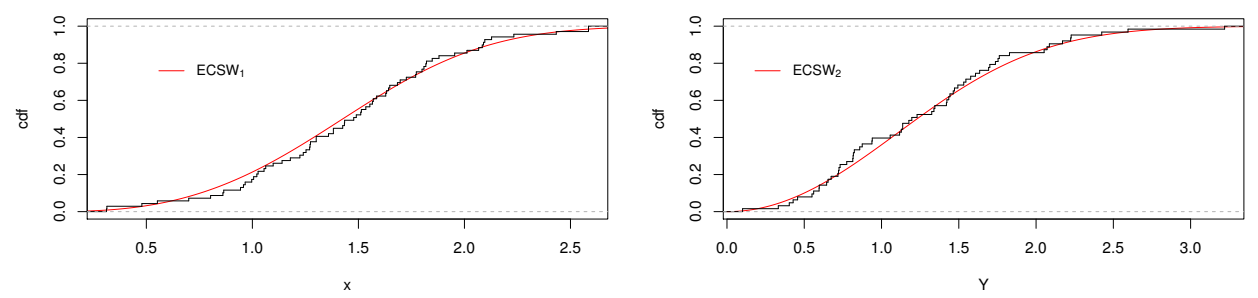

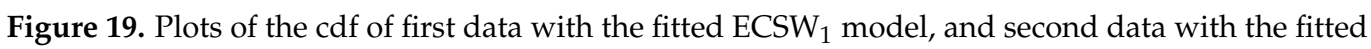
$\mathrm{ECSW}_{2}$ model.
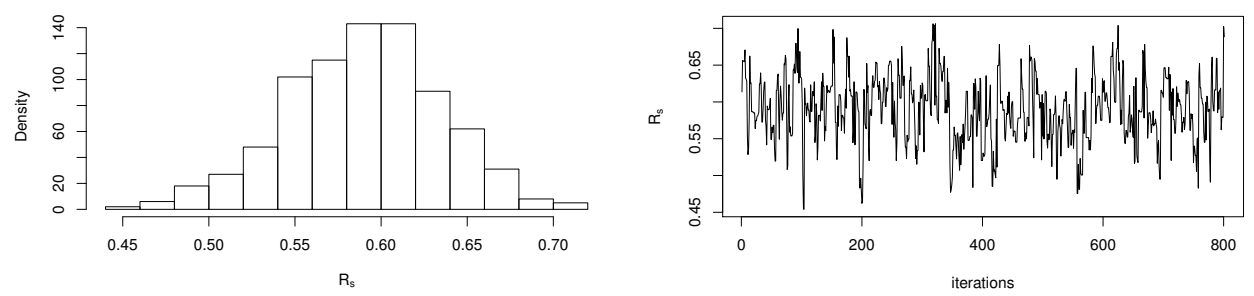

Figure 20. Plots of the histogram and estimated values of $R_{S}$ obtained from the Bayes estimates.

\section{Conclusions}

In this work, we proposed the new extended cosine-generated (ECSG) family of distributions. It contributed to the development of the trigonometric families by extending the famous sine-generated (SG) family, which has demonstrated a lot of success in recent years. Original and flexible distributions were derived, thanks to the joint oscillating nature of the involved trigonometric functions and the modulation of one additional parameter. Some important mathematical and statistical properties were derived, such as asymptotic results, quantile function, the series representation of the cumulative distribution and probability density functions, moments, moments of residual life, reliability parameter, and order statistics. Three special members were proposed and discussed, including the extended cosine Weibull (ECSW), extended cosine power (ECSP), and extended cosine generalized half-logistic (ECSGHL) distributions. The unknown parameters estimation was performed using the maximum likelihood estimation, least-square estimation, percentile estimation, and Bayes estimation under the SEL. All the proposed techniques provided a good result as examined by simulation studies of estimates' bias and mean square error. The stress-strength reliability studies from the ECSW distribution were discussed under common scale parameters and estimated using the BE with a gamma prior under the square error loss function (SEL), absolute error loss function, maximum a posteriori, general entropy loss function, and linear exponential loss function. A simulation was considered to study the Bayes estimates of the stress-strength parameter as discussed by the bias, mean square error, confidence interval, and coverage probability. Finally, three real data applications of the ECSG family were provided for illustration; one of them was stress- 
strength data discussed using the ECSW distribution. The real data studies showed the ECSG family's nice performance and illustrate that the ECSW model could be considered a good candidate for stress-strength reliability studies. Possible perspectives of the ECSG family were the construction of trigonometric regression models, skewed trigonometric distributions, and copulas functions. All these directions need more investigations, which we leave for future work. We hope that the new model will attract broader applications in various fields of study.

Author Contributions: Methodology, M.M., R.A.R.B., L.L., C.C., M.H.T., F.J. and M.E. All authors have read and agreed to the published version of the manuscript.

Funding: This work was supported by the funds of the Deanship of Scientific Research (DSR), King AbdulAziz University, Jeddah, under grant no. FP-042-43.

Institutional Review Board Statement: Not applicable.

Informed Consent Statement: Not applicable.

Data Availability Statement: Not applicable.

Acknowledgments: The authors express their gratitude to the referees for their insightful comments.

Conflicts of Interest: The authors declare that they have no conflict of interest.

\section{References}

1. Fisher, N.I. Statistical Analysis of Directional Data; University Press: Cambridge, UK, 1993.

2. $\quad$ Evans, M.; Hastings, N.; Peacock, B. Statistical Distributions; IOP Publishing: Bristol, UK, 2001.

3. Nadarajah, S.; Kotz, S. Beta trigonometric distributions. Port. Econ. J. 2006, 5, 207-224. [CrossRef]

4. Kent, J.T.; Tyler, D.E. Maximum likelihood estimation for the wrapped Cauchy distribution. J. Appl. Stat. 1988, 15, 247-254. [CrossRef]

5. Al-Faris, R.Q.; Khan, S. Sine square distribution: A new statistical model based on the sine function. J. Appl. Probab. Stat. 2008, 3, 163-173.

6. Souza, L.; Junior, W.; de Brito, C.; Chesneau, C.; Ferreira, T.; Soares, L. On the Sin-G class of distributions: Theory, model and application. J. Math. Model. 2019, 7, 357-379.

7. Kumar, D.; Singh, U.; Singh, S.K. A new distribution using sine function-its application to bladder cancer patients data. J. Stat. Appl. Probab. 2015, 4, 417.

8. Souza, L.; Junior, W.; de Brito, C.; Chesneau, C.; Ferreira, T.; Soares, L. General properties for the Cos-G Class of Distributions with Applications. Eurasian Bull. Math. 2019, 2, 63-79.

9. Chesneau, C.; Bakouch, H.S.; Hussain, T. A new class of probability distributions via cosine and sine functions with applications. Commun. Stat. Simul. Comput. 2019, 48, 2287-2300. [CrossRef]

10. Jamal, F.; Chesneau, C.; Bouali, D.; Ul Hassan, M. Beyond the Sin-G family: The transformed Sin-G family. PLoS ONE 2021, 16, e0250790. [CrossRef]

11. Mahmood, Z.; Chesneau, C.; Tahir, M. A new sine-G family of distributions: Properties and applications. Bull. Comput. Appl. Math. 2019, 7, 53-81.

12. Souza, L.; Junior, W.; de Brito, C.; Chesneau, C.; Fernandes, R.; Ferreira, T. Tan-G class of trigonometric distributions and its applications. Cubo 2021, 23, 1-20. [CrossRef]

13. Jamal, F; Chesneau, C. A new family of polyno-expo-trigonometric distributions with applications. Infin. Dimens. Anal. Quantum Probab. Relat. Top. 2019, 22, 1950027. [CrossRef]

14. Al-Babtain, A.A.; Elbatal, I.; Chesneau, C.; Elgarhy, M. Sine Topp-Leone-G family of distributions: Theory and applications. Open Phys. 2020, 18, 574-593. [CrossRef]

15. Jamal, F.; Chesneau, C.; Aidi, K. The sine extended odd Fréchet-G family of distribution with applications to complete and censored data. Math. Slovaca 2021, 71, 961-982. [CrossRef]

16. Bakouch, H.S.; Chesneau, C.; Leao, J. A new lifetime model with a periodic hazard rate and an application. J. Stat. Comput. Simul. 2018, 88, 2048-2065. [CrossRef]

17. Abate, J.; Choudhury, G.L.; Lucantoni, D.M.; Whitt, W. Asymptotic analysis of tail probabilities based on the computation of moments. Ann. Appl. Probab. 1995, 5, 983-1007. [CrossRef]

18. Gupta, R.C.; Gupta, P.L.; Gupta, R.D. Modeling failure time data by Lehman alternatives. Commun. Stat.-Theory Methods 1998, 27, 887-904. [CrossRef]

19. Poondi, K. A generalized probability density function for double-bounded random processes. J. Hydrol. 1980, 46, 79-88.

20. Gradshteyn, I.; Ryzhik, I.; Jeffrey, A.; Zwillinger, D. Table of Integrals, Series and Products, 7th ed.; Academic Press: New York, NY, USA, 2007. 
21. Kotz, S.; Pensky, M. The Stress-Strength Model and Its Generalizations: Theory and Applications; World Scientific: Singapore, 2003.

22. Guo, H.; Krishnamoorthy, K. New approximate inferential methods for the reliability parameter in a stress-strength model: The normal case. Commun. Stat.-Theory Methods 2004, 33, 1715-1731. [CrossRef]

23. Barbiero, A. Confidence intervals for reliability of stress-strength models in the normal case. Commun. Stat.-Simul. Comput. 2011, 40, 907-925. [CrossRef]

24. Baklizi, A.; El-Masri, A.E.Q. Shrinkage estimation of $\mathrm{P}(\mathrm{X}<\mathrm{Y})$ in the exponential case with common location parameter. Metrika 2004, 59, 163-171.

25. Asgharzadeh, A.; Valiollahi, R.; Raqab, M.Z. Estimation of the stress-Strength reliability for the generalized logistic distribution. Stat. Methodol. 2013, 15, 73-94. [CrossRef]

26. Krishnamoorthy, K.; Lin, Y. Confidence limits for stress-Strength reliability involving Weibull models. J. Stat. Plan. Inference 2010, 140, 1754-1764. [CrossRef]

27. Kundu, D.; Gupta, R.D. Estimation of $\mathrm{P}[\mathrm{Y}<\mathrm{X}]$ for Weibull distributions. IEEE Trans. Reliab. 2006, 55, $270-280$.

28. Asgharzadeh, A.; Valiollahi, R.; Raqab, M.Z. Stress-strength reliability of Weibull distribution based on progressively censored samples. SORT-Stat. Oper. Res. Trans. 2011, 35, 103-124.

29. Kundu, D.; Gupta, R.D. Estimation of P [Y < X] for generalized exponential distribution. Metrika 2005, 61, 291-308.

30. Nadarajah, S.; Bagheri, S.; Alizadeh, M.; Samani, E.B. Estimation of the stress strength parameter for the generalized exponentialPoisson distribution. J. Test. Eval. 2018, 46, 2184-2202. [CrossRef]

31. Shrahili, M.; Elbatal, I.; Muhammad, I.; Muhammad, M. Properties and applications of beta Erlang-truncated exponential distribution. J. Math. Comput. Sci. (JMCS) 2021, 22, 16-37. [CrossRef]

32. Muhammad, M.; Liu, L. A New Extension of the Generalized Half Logistic Distribution with Applications to Real Data. Entropy 2019, 21, 339. [CrossRef]

33. Muhammad, I.; Wang, X.; Li, C.; Yan, M.; Chang, M. Estimation of the Reliability of a Stress-Strength System from Poisson Half Logistic Distribution. Entropy 2020, 22, 1307. [CrossRef]

34. Arnold, B.C.; Balakrishnan, N.; Nagaraja, H.N. A First Course in Order Statistics; Society for Industrial and Applied Mathematics: Philadelphia, PA, USA, 1992; Volume 54.

35. Mächler, M. Arbitrarily Accurate Computation with R: The Rmpfr Package. Available online: https:/ / cran.r-project.org/web/ packages/Rmpfr/vignettes/Rmpfr-pkg.pdf (accessed on 1 October 2021).

36. Kantam, R.; Ramakrishna, V.; Ravikumar, M. Estimation and testing in type I generalized half logistic distribution. J. Mod. Appl. Stat. Methods 2013, 12, 22. [CrossRef]

37. Chen, M.H.; Shao, Q.M. Monte Carlo estimation of Bayesian credible and HPD intervals. J. Comput. Graph. Stat. 1999, 8, 69-92.

38. Metropolis, N.; Rosenbluth, A.W.; Rosenbluth, M.N.; Teller, A.H.; Teller, E. Equation of state calculations by fast computing machines. J. Chem. Phys. 1953, 21, 1087-1092. [CrossRef]

39. Hastings, W.K. Monte Carlo sampling methods using Markov chains and their applications. Biometrika 1970, 57, 97-109. [CrossRef]

40. Gelfand, A.E.; Smith, A.F. Sampling-based approaches to calculating marginal densities. J. Am. Stat. Assoc. 1990, 85, 398-409. [CrossRef]

41. Team, R.C. R: A Language and Environment for Statistical Computing; R Foundation for Statistical Computing: Vienna, Austria, 2019,

42. Hinkley, D. On quick choice of power transformation. J. R. Stat. Soc. Ser. C (Appl. Stat.) 1977, 26, 67-69. [CrossRef]

43. Seo, J.I.; Kang, S.B. Notes on the exponentiated half logistic distribution. Appl. Math. Model. 2015, 39, 6491-6500. [CrossRef]

44. Bagheri, S.; Samani, E.B.; Ganjali, M. The generalized modified Weibull power series distribution: Theory and applications. Comput. Stat. Data Anal. 2016, 94, 136-160. [CrossRef]

45. Muhammad, M. Poisson-odd generalized exponential family of distributions: Theory and applications. Hacet. J. Math. Stat. 2016, 47, 1652-1670. [CrossRef]

46. Sarhan, A.M.; Abd EL-Baset, A.A.; Alasbahi, I.A. Exponentiated generalized linear exponential distribution. Appl. Math. Model. 2013, 37, 2838-2849. [CrossRef]

47. Torabi, H.; Hedesh, N.M. The gamma-uniform distribution and its applications. Kybernetika 2012, 48, 16-30.

48. Sarhan, A.M.; Kundu, D. Generalized linear failure rate distribution. Commun. Stat.-Theory Methods 2009, 38, 642-660. [CrossRef]

49. Lee, C.; Famoye, F.; Olumolade, O. Beta-Weibull distribution: Some properties and applications to censored data. J. Mod. Appl. Stat. Methods 2007, 6, 17. [CrossRef]

50. Carrasco, J.M.; Ortega, E.M.; Cordeiro, G.M. A generalized modified Weibull distribution for lifetime modeling. Comput. Stat. Data Anal. 2008, 53, 450-462. [CrossRef]

51. Lai, C.; Xie, M.; Murthy, D. A modified Weibull distribution. IEEE Trans. Reliab. 2003, 52, 33-37. [CrossRef]

52. Mahmoud, M.A.; Alam, F.M.A. The generalized linear exponential distribution. Stat. Probab. Lett. 2010, 80, 1005-1014. [CrossRef]

53. Silva, G.O.; Ortega, E.M.; Cordeiro, G.M. The beta modified Weibull distribution. Lifetime Data Anal. 2010, 16, 409-430. [CrossRef]

54. Cordeiro, G.M.; de Andrade, T.A.; Bourguignon, M.; Gomes-Silva, F. The exponentiated generalized standardized half-logistic distribution. Int. J. Stat. Probab. 2017, 6, 24-42. [CrossRef]

55. Abdel-Hamid, A.H. Properties, estimations and predictions for a Poisson-half-logistic distribution based on progressively type-II censored samples. Appl. Math. Model. 2016, 40, 7164-7181. [CrossRef]

56. Olapade, A. The type I generalized half logistic distribution. J. Iran. Stat. Soc. 2014, 13, 69-82. 
57. Jose, J.K.; Manoharan, M. Beta half logistic distribution-A new probability model for lifetime data. J. Stat. Manag. Syst. 2016, 19, 587-604. [CrossRef]

58. Muhammad, M.; Yahaya, M.A. The half logistic-Poisson distribution. Asian J. Math. Appl. 2017, 2017.

59. Muhammad, M. Generalized half-logistic Poisson distributions. Commun. Stat. Appl. Methods 2017, 24, 353-365. [CrossRef]

60. Muhammad, M.; Liu, L. A New Three Parameter Lifetime Model: The Complementary Poisson Generalized Half Logistic Distribution. IEEE Access 2021, 9, 60089-60107. [CrossRef]

61. Aarset, M.V. How to identify a bathtub hazard rate. IEEE Trans. Reliab. 1987, 36, 106-108. [CrossRef]

62. Nichols, M.D.; Padgett, W. A bootstrap control chart for Weibull percentiles. Qual. Reliab. Eng. Int. 2006, 22, 141-151. [CrossRef]

63. Bader, M.; Priest, A. Statistical aspects of fibre and bundle strength in hybrid composites. In Progress in Science and Engineering of Composites; NHPC: Edmonton, AB, Canada, 1982; pp. 1129-1136.

64. Raqab, M.Z.; Madi, M.T.; Kundu, D. Estimation of $\mathrm{P}(\mathrm{Y}<\mathrm{X})$ for the three-parameter generalized exponential distribution. Commun. Stat. Methods 2008, 37, 2854-2864. 